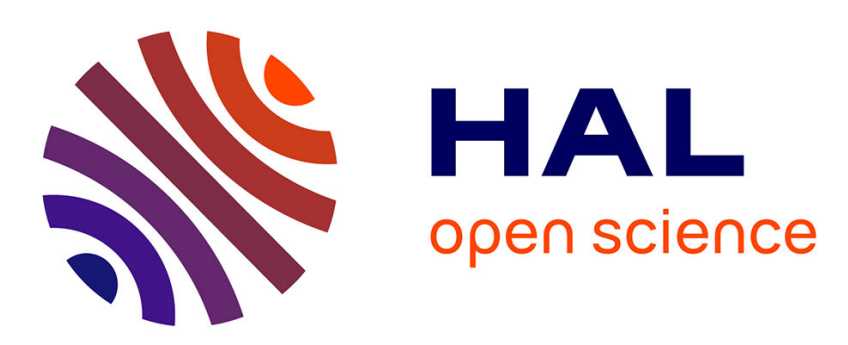

\title{
Trailing-edge noise prediction by solving Helmholtz equation with stochastic source term
}

Ali Hussain Kadar, Sophie Le Bras, Hadrien Bériot, Wim de Roeck, Wim Desmet, Christophe Schram

\section{- To cite this version:}

Ali Hussain Kadar, Sophie Le Bras, Hadrien Bériot, Wim de Roeck, Wim Desmet, et al.. Trailingedge noise prediction by solving Helmholtz equation with stochastic source term. AIAA Journal, 2021, 10.2514/1.J060388 . hal-03495094

\section{HAL Id: hal-03495094 \\ https://hal.science/hal-03495094}

Submitted on 20 Dec 2021

HAL is a multi-disciplinary open access archive for the deposit and dissemination of scientific research documents, whether they are published or not. The documents may come from teaching and research institutions in France or abroad, or from public or private research centers.
L'archive ouverte pluridisciplinaire HAL, est destinée au dépôt et à la diffusion de documents scientifiques de niveau recherche, publiés ou non, émanant des établissements d'enseignement et de recherche français ou étrangers, des laboratoires publics ou privés. 


\title{
Trailing-edge noise prediction by solving Helmholtz equation with stochastic source term
}

\author{
Ali Hussain Kadar*, Sophie Le Bras ${ }^{\dagger}$ and Hadrien Bériot ${ }^{\ddagger}$ \\ Siemens Industry Software N.V., Leuven, 3001, Belgium \\ Wim De Roeck ${ }^{\S}$ and Wim Desmet ${ }^{\text {II }}$ \\ KU Leuven, Leuven, 3001, Belgium \\ Christophe Schram" \\ von Kármán Institute for Fluid Dynamics, Rhode-St-Genèse, 1640, Belgium
}

A numerical approach to predict broadband trailing-edge noise for low Mach number flows is presented. It is based on the combination of a Helmholtz solver to propagate sound waves with a sound source term computed stochastically. The sound propagation is performed in the frequency domain using a high-order finite element solver. The stochastic approach is based on a Random Particle-Mesh method. The performance of this numerical approach is first examined for a Gaussian source. The numerical approach is then applied to a NACA0012 airfoil for flow Mach numbers of 0.11 and 0.16 and to a controlled-diffusion airfoil for a Mach number of 0.047. The predicted sound levels are compared with experimental data and acoustic results obtained from the Acoustic Perturbations Equations. The mean flow does not significantly modify the acoustic propagation. Using a no-flow propagation model like the Helmholtz equation is therefore a valid approach for low Mach numbers. The reduced computational cost of a Helmholtz calculation, together with the speed of the RPM turbulence synthesis, allows for fast predictions. While this approach provides relative assessments between different configurations (in particular the spectrum shape), it often requires the inclusion of calibration factors determined from reference measurement or numerical data.

\section{Nomenclature}

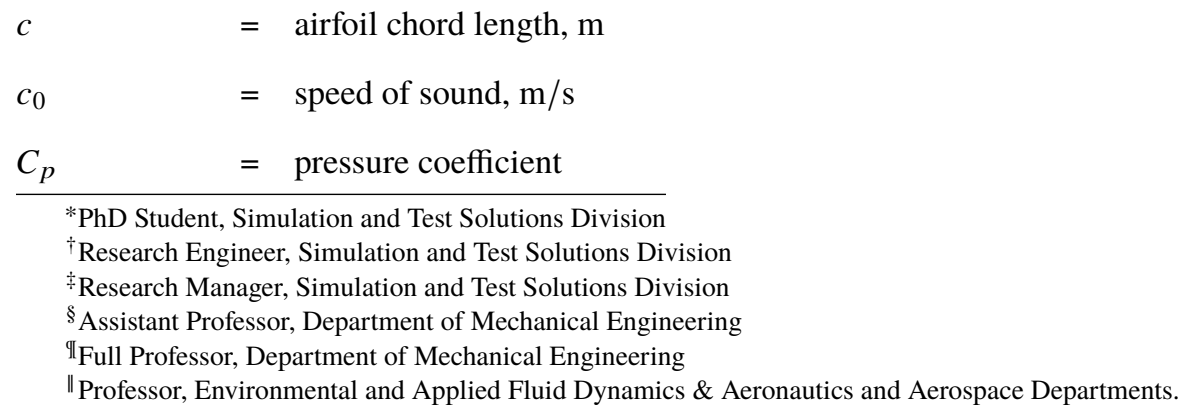




\begin{tabular}{|c|c|}
\hline$d_{\lambda}$ & $=$ degrees of freedom per acoustic wavelength \\
\hline$E_{T}$ & $=$ finite-element target accuracy \\
\hline$f$ & $=$ frequency, $\mathrm{Hz}$ \\
\hline$G$ & $=$ spatial filter function \\
\hline$\widetilde{G}$ & $=$ Green's function \\
\hline$h$ & $=$ grid spacing \\
\hline$k$ & $=$ turbulent kinetic energy, $\mathrm{m}^{2} / \mathrm{s}^{2}$ \\
\hline$k_{\omega}$ & $=$ acoustic wavenumber $\left(k_{\omega}=\omega / c_{0}\right), 1 / \mathrm{m}$ \\
\hline$k_{c}$ & $=$ chord-based Helmholtz number \\
\hline$L$ & $=$ airfoil span, $\mathrm{m}$ \\
\hline$L_{x}, L_{y}$ & $=$ size of the RPM domain, $\mathrm{m}$ \\
\hline$L^{\prime}$ & $=$ Lamb vector, $\mathrm{m} / \mathrm{s}^{2}$ \\
\hline$M$ & $=$ Mach number \\
\hline$n$ & $=$ unit outward normal vector \\
\hline$N$ & $=$ number of particles in RPM domain \\
\hline$N_{\mathrm{ppc}}$ & $=$ number of particles per RPM grid cell \\
\hline$p_{\text {FEM }}, p_{\text {source }}$ & $=$ finite-element polynomial orders \\
\hline$p^{\prime}, p_{\text {ref }}$ & $=$ acoustic pressure and reference acoustic pressure, $\mathrm{Pa}$ \\
\hline$q$ & $=$ finite-element test function \\
\hline$R e_{\mathrm{c}}$ & $=$ Reynolds number based on the chord length \\
\hline$u_{\infty}$ & $=$ free-stream velocity, $\mathrm{m} / \mathrm{s}$ \\
\hline $\boldsymbol{u}_{0}, \omega_{0}$ & $=$ mean flow velocity, $\mathrm{m} / \mathrm{s}$ and vorticity, $1 / \mathrm{s}$ \\
\hline$u^{\prime}, \omega^{\prime}$ & $=$ fluctuating velocity, $\mathrm{m} / \mathrm{s}$ and vorticity, $1 / \mathrm{s}$ \\
\hline$W$ & $=$ stochastic field \\
\hline$y^{+}$ & $=$non-dimensional distance from wall \\
\hline$\alpha$ & $=$ airfoil angle of attack \\
\hline$\delta$ & $=$ Dirac delta function \\
\hline$\epsilon_{i j}$ & $=$ Levi-Civita symbol \\
\hline$\lambda$ & $=$ acoustic wavelength, $\mathrm{m}$ \\
\hline$\Lambda$ & $=$ integral length scale of turbulence, $\mathrm{m}$ \\
\hline$v^{*}$ & $=$ Courant-Friedrichs-Lewy number \\
\hline$\omega$ & $=$ angular frequency, $1 / \mathrm{s}$ \\
\hline
\end{tabular}




$\begin{array}{ll}\omega_{t} & =\text { specific dissipation rate, } 1 / \mathrm{s} \\ \Omega_{0} & =\text { sound source region } \\ \Omega & =\text { acoustic propagation domain } \\ \psi^{\prime} & =\text { fluctuating stream function, } \mathrm{m}^{2} / \mathrm{s} \\ \rho_{0} & =\text { mean flow density, } \mathrm{kg} / \mathrm{m}^{3} \\ \tau & =\text { integral time scale of turbulence, } \mathrm{s}\end{array}$

\section{Introduction}

$\mathrm{T}$ He scattering of turbulent boundary layer velocity fluctuations at the trailing edge of airfoils or blades can be the dominant source of noise of low-speed cooling fans, wind turbines or aircraft lifting surfaces [1, 2]. This mechanism remains even in the absence of incoming free stream turbulence. An accurate prediction of trailing-edge noise is therefore required in order to design quieter solutions. Hybrid Computational AeroAcoustic (CAA) strategies, where the sound source calculation is decoupled from the sound propagation, have been successfully applied to predict trailing-edge noise, in particular when high-fidelity Computational Fluid Dynamics (CFD) techniques such as large-eddy simulation are used to calculate the equivalent sources [3-5]. However, despite continuous progress in high-performance computing, high-fidelity CFD techniques remain computationally expensive and often inappropriate for industrial design through optimization. This is mainly due to the high computational costs associated with the temporal and spatial resolution of the unsteady turbulent flow scales generating noise.

Stochastic methods have been developed as an alternative to scale-resolved simulations, where the turbulent velocity fluctuations are synthesized instead of being resolved. This approach rests upon the theoretical result that the sound power spectrum depends solely on the two-point space-time correlations of the equivalent source field [6-8] and on the Green's function of the scattering problem at stake. Since those two-point space-time correlations can be modelled, via some physical assumptions [9], using data provided by relatively inexpensive Reynolds-Averaged Navier-Stokes (RANS) simulations, the stochastic approach opens the possibility to obtain noise predictions for a fraction of the numerical cost associated with scale-resolved CFD techniques.

Two main approaches have been developed for the synthesis of stochastic turbulence, based on Fourier modes [10-12] or digital filtering. Both approaches have pros and cons, and several improvements have been developed over the years to overcome their respective shortcomings [13-15]. While the Fourier mode approach is recognized for its ability to control the spectral content of the turbulent velocity, its computational and memory cost can be prohibitive. The digital filtering approach is known for its ability to account more easily for non-uniformities of the turbulence statistics. It also accurately synthetizes the two-point correlation tensor of homogeneous isotropic turbulence. Like the Fourier mode approach, it can suffer from long run times and large memory usage, especially when velocity fluctuations have to be 
synthetized and stored in 3-D over long physical times. The present work follows the digital filtering approach pioneered by Careta $e t$ al. [16] to generate a two-dimensional divergence-free isotropic turbulent velocity field, and by Klein $e t$ al. [17] who developed a digital-filter-based method to generate inflow turbulence in scale-resolved CFD simulations. Based on those works, Ewert et al. [6, 18-20] introduced the Random Particle-Mesh (RPM) method, a divergence-free stochastic approach accounting for flow convection effects. Applied by several research groups for different applications including airfoil noise [21, 22], jet noise [23], fan noise [24] or HVAC duct noise [25], the RPM technique permits relative assessments between different configurations at moderate computational costs. For trailing-edge noise problems, it can even provide absolute levels via some calibration of the sound levels using reference measurement or numerical data [26].

Concerning the propagation of the sound waves with sound sources generated stochastically, the literature mostly reports about time-based acoustic solvers, discretizing the Linearized Euler Equations (LEE) or the Acoustic Perturbation Equations (APE) [20, 22, 27]. While this fits the time-marching nature of the RPM source generation, the stability requirements imposed by the Courant-Friedrichs-Lewy (CFL) criterion for explicit time-discretization schemes [28] can severely limit the maximum admissible time step. As a result, the total run time and amount of data can become unnecessarily large compared to what the Nyquist sampling rule alone would impose for the frequencies of interest. An alternative strategy consists in simulating the sound propagation in the frequency domain. For instance, Cozza et al. [21] proposed a frequency-domain approach based on the resolution of the APE with a source term defined by RPM. Casalino et al. [14] derived an aeroacoustic approach based on a linearized Howe equation for sound propagation combined with a sound source term defined using Fourier modes. In the frequency domain, the acoustic equations can be robustly solved using direct solvers such as the MUltifrontal Massively Parallel Solver MUMPS [29]. A limiting factors remains however the massive amounts of memory required for the resolution.

A different approach is proposed in this work, motivated by the fairly low Mach numbers of many configurations of interest, such as wind turbines or low-speed cooling fans, for which the effects of acoustic refraction and convection on noise propagation can be neglected at first order. In such conditions, the acoustic propagation equations can be simplified to form in the frequency domain a Helmholtz equation, assuming a quiescent and uniform propagation medium. This scalar acoustic propagation equation can be solved quite efficiently via Finite Element Methods (FEM) or Boundary Element Methods (BEM), having a much reduced memory footprint compared to the APE system. A high-order unstructured FEM approach [30] is used here. This paper addresses the applicability, numerical efficiency and accuracy of this coupling strategy, for a source synthesis performed on the basis of two-dimensional RANS simulations and an acoustic model accounting for three-dimensional propagation. The effects of the mean flow are assessed by comparing sound predictions obtained from the Helmholtz solver and from the APE. This simulation procedure is meant to yield a favorable trade-off between numerical accuracy and numerical cost for configurations that exhibit a statistical homogeneity in a spanwise direction. 
This paper is organized as follows. In section II, the Helmholtz-based aeroacoustic approach is presented together with the FEM solver and the source interpolation techniques. In section III, the performance of the numerical approach is first evaluated by simulating the sound generated by a Gaussian-distributed scalar source [31]. The methodology is then applied to predict the sound field radiated from the trailing-edge of a NACA0012 airfoil in section IV. To investigate the effects of the mean flow, numerical simulations are performed for two flow Mach numbers using APE and Helmholtz FEM solvers. In section V, the RPM-Helmholtz approach is applied to a Controlled-Diffusion (CD) airfoil for a low Mach number flow $M=0.047$ [32, 33]. The results are compared with numerical [34] and experimental [32, 33] data from the literature. A parametric study is conducted in order to examine the sensitivity of the acoustic results to the definition of the sound source term and the acoustic grid.

\section{Computational approach}

The present numerical approach comprises four steps. The turbulence statistics are first calculated analytically or by performing a RANS simulation. The sound sources are then computed using a time-domain RPM approach. In a third step, the sound sources are converted into frequency-domain sources using a fast Fourier transform and interpolated on the unstructured mesh used for the acoustic propagation in the last step. In this work, the resolution of the Helmholtz equation is carried out using high-order FEM [30].

\section{A. Acoustic propagation model}

\section{Acoustic Perturbation Equations (APE-4)}

The sound propagation model used in this study is derived from the Acoustic Perturbation Equations APE-4 [27]. The APE-4 are obtained from the Linearized Euler Equations (LEE) excluding the non-acoustic modes. They describe the propagation of sound waves taking into account the convection and the refraction effects of a non-uniform mean flow. For a source term $\left(q_{c}^{\prime}, \boldsymbol{q}_{m}^{\prime}\right)$, the APE-4 write as:

$$
\left\{\begin{array}{l}
\frac{\partial p^{\prime}}{\partial t}+c_{0}^{2} \nabla \cdot\left(\rho_{0} \boldsymbol{u}^{\prime}+\frac{p^{\prime}}{c_{0}^{2}} \boldsymbol{u}_{0}\right)=q_{c}^{\prime}, \\
\frac{\partial \boldsymbol{u}^{\prime}}{\partial t}+\nabla\left(\boldsymbol{u}_{0} \cdot \boldsymbol{u}^{\prime}\right)+\nabla\left(\frac{p^{\prime}}{\rho_{0}}\right)=\boldsymbol{q}_{m}^{\prime}
\end{array}\right.
$$

where the variables $p^{\prime}$ and $\boldsymbol{u}^{\prime}$ are the pressure and velocity perturbations, $c_{0}$ is the speed of sound, and $\rho_{0}$ and $\boldsymbol{u}_{0}$ are the mean flow density and velocity. For trailing-edge noise problems, the source term can be defined as follows:

$$
\left\{\begin{array}{l}
q_{c}^{\prime}=0 \\
\boldsymbol{q}_{m}^{\prime}=-\boldsymbol{L}^{\prime}
\end{array}\right.
$$


where $L^{\prime}$ is called the Lamb vector, a vorticity-based source term also found in the acoustic analogies of Powell and Howe $[35,36]$. It is defined as:

$$
L^{\prime}=(\omega \times \boldsymbol{u})^{\prime}=\omega_{0} \times u^{\prime}+\omega^{\prime} \times u_{0}+\left(\omega^{\prime} \times u^{\prime}\right)^{\prime} \approx \omega_{0} \times u^{\prime}+\omega^{\prime} \times u_{0}
$$

where $\omega^{\prime}$ is the fluctuating vorticity vector, and $\omega_{0}$ is the mean vorticity vector. Following [20, 37], the non-linear term $\left(\boldsymbol{\omega}^{\prime} \times \boldsymbol{u}^{\prime}\right)^{\prime}$ in Eq. (5) is neglected.

\section{Acoustic propagation in the frequency domain for low Mach number flows}

For low Mach number flows, when the effects of the mean-flow convection and refraction can be neglected [38], the APE system can be simplified. Further assuming a uniform density $\rho_{0}$ and combining the time derivative of Eq. (1) with the divergence of Eq. (2), an inhomogeneous scalar wave equation is obtained:

$$
\frac{\partial^{2} p^{\prime}}{\partial t^{2}}-c_{0}^{2} \nabla^{2} p^{\prime}=\frac{\partial q_{c}^{\prime}}{\partial t}-\rho_{0} c_{0}^{2} \nabla \cdot \boldsymbol{q}_{m}^{\prime}
$$

In order to perform the acoustic propagation in the frequency domain, Equation (6) is transformed to the spectral space by applying a Fourier transform. The Fourier transform in time of a signal $\phi(x, t)$ is defined as:

$$
\mathcal{F}[\phi(\boldsymbol{x}, t)]=\hat{\phi}(\boldsymbol{x}, \omega)=\int_{-\infty}^{\infty} \phi(\boldsymbol{x}, t) e^{-\mathrm{i} \omega t} \mathrm{~d} t
$$

where $\omega$ is the angular frequency. It yields the following inhomogeneous Helmholtz equation:

$$
\nabla^{2} \hat{p}+k_{\omega}^{2} \hat{p}=-\mathrm{i} \frac{k_{\omega}}{c_{0}} \widehat{q}_{c}+\rho_{0} \nabla \cdot \widehat{\boldsymbol{q}}_{m}
$$

where $k_{\omega}=\omega / c_{0}$ is the wavenumber and $\hat{p}, \widehat{q}_{c}$ and $\widehat{\boldsymbol{q}}_{m}$ are the Fourier coefficients of variables $p^{\prime}, q_{c}^{\prime}$ and $\boldsymbol{q}_{m}^{\prime}$ respectively.

\section{Resolution of a Helmholtz problem using a Finite Element Method}

The inhomogeneous Helmholtz equation (8) is solved using a high-order Finite-Element Method (FEM) [30]. A sketch of the acoustic propagation problem applied to an airfoil is shown in Fig. 1, where $\Omega$ is the computational domain, $\partial \Omega_{s}$ is the airfoil scattering surface which is modelled as a slip wall, and $\partial \Omega_{\infty}$ is the external surface where Non-Reflecting Boundary Conditions (NRBC) are prescribed. The Helmholtz problem results in the following set of 
equations:

$$
\left\{\begin{array}{l}
\nabla^{2} \hat{p}+k_{\omega}^{2} \hat{p}=-\mathrm{i} \frac{k_{\omega}}{c_{0}} \widehat{q}_{c}+\rho_{0} \nabla \cdot \widehat{\boldsymbol{q}}_{m} \quad \forall \boldsymbol{x} \in \Omega, \\
\nabla \hat{p} \cdot \boldsymbol{n}=0 \quad \forall \boldsymbol{x} \in \partial \Omega_{s}, \\
\mathrm{NRBC} \quad \forall \boldsymbol{x} \in \partial \Omega_{\infty},
\end{array}\right.
$$

where $\boldsymbol{n}$ is the unit normal vector on surface $\partial \Omega_{s}$ pointing out of domain $\Omega$.

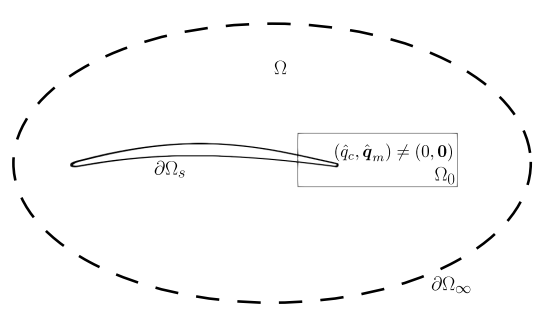

Fig. 1 Sketch of the computational domain used for the sound propagation using FEM.

The Helmholtz problem is solved using a Galerkin finite-element approach. The variational formulation is defined as:

$$
-\int_{\Omega} \nabla \bar{q} \cdot \nabla \hat{p} \mathrm{~d} \Omega+k_{\omega}^{2} \int_{\Omega} \bar{q} \hat{p} \mathrm{~d} \Omega+\int_{\partial \Omega} \bar{q}(\nabla \hat{p} \cdot \boldsymbol{n}) \mathrm{d} \Gamma=\int_{\Omega} \bar{q}\left(-\mathrm{i} \frac{k_{\omega}}{c_{0}} \widehat{q}_{c}+\rho_{0} \nabla \cdot \widehat{\boldsymbol{q}}_{m}\right) \mathrm{d} \Omega,
$$

where $q$ is the test function and the overbar - is the complex conjugate operator. In order to avoid calculating the divergence of the source term $\widehat{\boldsymbol{q}}_{m}$, the second term of the right-hand side of Eq. (12) is integrated by parts yielding:

$$
\int_{\Omega} \bar{q}\left(\rho_{0} \nabla \cdot \widehat{\boldsymbol{q}}_{m}\right) \mathrm{d} \Omega=\int_{\partial \Omega} \rho_{0}\left(\widehat{\boldsymbol{q}}_{m} \cdot \boldsymbol{n}\right) \bar{q} \mathrm{~d} \Gamma-\int_{\Omega} \rho_{0} \nabla \bar{q} \cdot \widehat{\boldsymbol{q}}_{m} \mathrm{~d} \Omega
$$

For trailing-edge noise problems, the source term in the Fourier space is $(0,-\widehat{\boldsymbol{L}})$. As the values of $\widehat{\boldsymbol{L}}$ are computed in a bounded region $\Omega_{0}$ in the vicinity of the trailing-edge, it is null on the external surface $\partial \Omega_{\infty}$. On the scattering surface $\partial \Omega_{s}$, the Lamb vector vanishes as the present stochastic sound model imposes zero-velocity fluctuation at the solid walls. In this case, the variational formulation (12) becomes:

$$
-\int_{\Omega} \nabla \bar{q} \cdot \nabla \hat{p} \mathrm{~d} \Omega+k_{\omega}^{2} \int_{\Omega} \bar{q} \hat{p} \mathrm{~d} \Omega+\int_{\partial \Omega_{\infty}} \bar{q}(\nabla \hat{p} \cdot \boldsymbol{n}) \mathrm{d} \Gamma=\int_{\Omega} \rho_{0} \nabla \bar{q} \cdot \widehat{\boldsymbol{L}} \mathrm{d} \Omega
$$

At the boundary $\partial \Omega_{\infty}$, a Perfectly Matched Layer (PML) is introduced in order to efficiently absorb the outgoing waves. We use here the locally-conformal implementation described in [39]. The latter is equipped with the parameter-free unbounded absorbing function from [40].

Equation (12) is discretized using a high-order finite-element method [41], named $p$-FEM. The domain $\Omega$ is partitioned into a set of non-overlapping triangular elements. In each element, the numerical solution is made up of high-order $H^{1}$-conforming hierarchical polynomial shape functions [41]. The $p$-FEM approach is less sensitive 
to dispersion errors and has shown to provide substantial reductions in memory and CPU time when compared to conventional low-order FEM on a variety of Helmholtz problems [30, 42]. In this work, a $p$-adaptive solution is used for improved efficiency. We resort to the a priori error indicator proposed in [43], which adjusts automatically the edge and face orders across the mesh so as to achieve a given user-defined $L^{2}$-error target accuracy $E_{T}$. The orders are here defined to be in the range $p_{\text {FEM }} \in[2,10]$. While the conventional strategy consists in creating a different mesh for each frequency of interest, this adaptive approach allows computing solutions seamlessly over a broad frequency range based on a single input mesh. The size of the largest mesh element is chosen to satisfy $p_{\text {FEM }} \leq 10$.

The sound source terms $\boldsymbol{q}_{m}^{\prime}$ and $q_{c}^{\prime}$ are calculated in the time domain using a stochastic approach presented in section II.B. In order to obtain statistically converged sound spectra, the source time signal $q_{c}^{\prime}\left(\right.$ resp. $\left.\boldsymbol{q}_{m}^{\prime}\right)$, computed over a period $T$, is divided into $N_{\text {seg }}$ segments whose length $T_{\text {seg }}$ is determined based on the desired minimum frequency and spectral resolution. A number of 30 segments leads to satisfactory results for the applications presented in this paper. The statistical convergence of the acoustic results with respect to the number of segments $N_{\text {seg }}$ is discussed in appendix VI.A. For each segment, the sound sources $\widehat{q}_{c}$ (resp. $\widehat{\boldsymbol{q}_{m}}$ ) in the frequency domain are evaluated at the nodes of the RPM grid using a Fast Fourier Transform (FFT) algorithm combined with a Hanning window to minimize spectral leakage. In order to account for the amplitude reduction due to the application of the Hanning window, the Fourier coefficients are multiplied by a correction factor of $\sqrt{8 / 3}$ in order to preserve the energy of the signal. The Fourier coefficients are then interpolated on the FEM mesh. Each time segment then leads to a different right-hand-side for the finite element linear system. A FEM simulation is then carried out for each segment and each frequency of interest. The values of $|\hat{p}|^{2}$ obtained over the $N_{\text {seg }}$ segments are then averaged to compute the power spectral density $S_{p p}$.

As reported in the literature [25,44], the sound source interpolation onto the acoustic mesh deserves some care in order to minimize the production of spurious noise. For the mapping approach chosen here, two continuous representations are needed, one for the RPM grid and one for the acoustic grid. On the structured RPM grid, on the one hand, a $C^{2}$-continuous cubic spline interpolation is used. On the acoustic mesh, on the other hand, a piecewise continuous polynomial approximation of order $p_{\text {source }}=10$ is constructed on each triangle, using the Lagrange shape functions with optimal node distribution from [45]. The latter is obtained by querying the source strength $\widehat{q}_{c}$ (or $\widehat{\boldsymbol{q}}_{m}$ ) from the RPM cubic spline representation at each acoustic triangular high-order source node location. The advantage of this approach is that it allows to better control the quadrature error, by consistently integrating the chosen acoustic source representation. In this paper, a quadrature rule of order $\left(p_{\mathrm{FEM}}-1+p_{\text {source }}\right)$ is used to evaluate the right-hand-side integral in Equation (14).

\section{B. Sound source model}

In order to compute the sound source term $\boldsymbol{L}^{\prime}$ given in Eq. (5), the mean velocity $\boldsymbol{u}_{0}$ and vorticity $\boldsymbol{\omega}_{0}$ can be prescribed analytically or from a RANS simulation. The fluctuating variables $\boldsymbol{u}^{\prime}$ and $\boldsymbol{\omega}^{\prime}$ are synthetized by a RPM 
method.

\section{Random Particle-Mesh method}

The RPM method is a stochastic approach that generates turbulent velocity fluctuations $\boldsymbol{u}^{\prime}$ from prescribed turbulence statistics in a given sound source region $\Omega_{0}[18,20]$. In order to obtain a divergence-free velocity field, the fluctuations $\boldsymbol{u}^{\prime}$ are computed from the curl of a perturbation stream function $\psi^{\prime}=\left[\psi_{1}^{\prime}, \psi_{2}^{\prime}, \psi_{3}^{\prime}\right]^{T}$. At a given position $\boldsymbol{x}$ in domain $\Omega_{0}$, the stream function component $\psi_{i}^{\prime}$ is obtained from the spatial convolution of a stochastic noise field $W_{i}$ with a filter kernel $G$ that defines the spatial properties of the turbulence:

$$
\psi_{i}^{\prime}(\boldsymbol{x}, t)=\int_{\Omega_{0}} G\left(\boldsymbol{x}-\boldsymbol{x}^{\prime}\right) W_{i}\left(\boldsymbol{x}^{\prime}, t\right) \mathrm{d} \boldsymbol{x}^{\prime},
$$

The stream function field $\psi_{i}^{\prime}$ can be seen as the weigthed sum of random contributions $W_{i}$ carried by particles located at positions $\boldsymbol{x}^{\prime}$ in domain $\Omega_{0}$. The filter $G$ is designed from an analytical energy spectrum $E$ of the turbulence [46]. Several spectra $E$ are found in the literature, such as the Gaussian, the Liepmann or the von Kármán spectra [46]. In this work, a Gaussian energy spectrum is chosen for its simplicity of implementation and for its low computational cost as compared to other spectra [46]. Under the assumption of locally homogeneous and isotropic turbulence, the Gaussian filter is defined as:

$$
G\left(\boldsymbol{x}-\boldsymbol{x}^{\prime}\right)=A\left(\boldsymbol{x}^{\prime}\right) \exp \left(\frac{-\pi\left|\boldsymbol{x}-\boldsymbol{x}^{\prime}\right|^{2}}{2 \Lambda\left(\boldsymbol{x}^{\prime}\right)^{2}}\right) \quad \text { with } \quad A\left(\boldsymbol{x}^{\prime}\right)=\sqrt{\frac{2 \rho_{0}\left(\boldsymbol{x}^{\prime}\right) k\left(\boldsymbol{x}^{\prime}\right)}{3 \pi \Lambda\left(\boldsymbol{x}^{\prime}\right)}}
$$

where $A$ is the filter amplitude, $\Lambda$ is the integral length scale of the turbulence, $k$ is the three-dimensional kinetic energy and $\rho_{0}$ is the mean flow density. The stochastic field $W_{i}$ in Eq. (15) satisfies the following properties:

$$
\begin{aligned}
<W_{i}\left(\boldsymbol{x}^{\prime}, t\right)> & =0, \\
<W_{i}\left(\boldsymbol{x}^{\prime}, t\right) W_{i}\left(\boldsymbol{x}^{\prime}+\boldsymbol{r}, t+s\right)> & =\rho_{0}\left(\boldsymbol{x}^{\prime}\right)^{-1} \delta\left(\boldsymbol{r}-s \boldsymbol{u}_{0}\left(\boldsymbol{x}^{\prime}\right)\right) \exp \left(-\frac{s}{\tau\left(\boldsymbol{x}^{\prime}\right)}\right),
\end{aligned}
$$

where $\tau$ is the integral time scale of the turbulence, $\boldsymbol{r}$ and $s$ are the spatial and temporal separations, the operator $\langle\cdot>$ is the time average and $\delta$ is the Dirac delta function. Assuming frozen turbulence, the time scale $\tau$ is infinite. For evolving turbulence, the exponential term in Eq. (18) prescribes the time-decorrelation. In this case, standard Langevin equations, originally designed to model Brownian motion, are solved to model the temporal properties of the stochastic fields $W_{i}$ [46]. Finally, the velocity and vorticity fluctuations $\boldsymbol{u}^{\prime}$ and $\omega^{\prime}$ are computed from the stream function $\psi^{\prime}$ as $\boldsymbol{u}^{\prime}=\nabla \times \psi^{\prime}$ and $\omega^{\prime}=\nabla \times \boldsymbol{u}^{\prime}$.

The mean flow values $\rho_{0}, \boldsymbol{u}_{0}, k, \Lambda$ and $\tau$ are obtained analytically or from a preliminary RANS computation. For RANS simulations based on SST $k-\omega$ turbulence model [47], the values of $\Lambda$ and $\tau$ can be obtained from the RANS 
energy $k$ and specific dissipation rate $\omega_{t}$ as:

$$
\Lambda=l_{\text {factor }} \frac{\sqrt{k}}{\omega_{t}} \quad \text { and } \quad \tau=\frac{2}{\beta C_{0} \omega_{t}}
$$

where $l_{\text {factor }}, C_{0}$ and $\beta=0.09$ are constant parameters. There is no concensus on the choice of the parameters $l_{\text {factor }}$ and $C_{0}$. The constant $l_{\text {factor }}$ is often equal to 6 [48], a value estimated from a modified von Kármán spectrum [12]. The choice of $l_{\text {factor }}$ and its influence on the acoustic results is discussed in section V.B.7. The empirical parameter is found to vary between 1 and 4 . The value $C_{0}=2.1$ is commonly used in the literature [49].

\section{Numerical discretization}

In this study, the numerical discretization of the RPM method follows the Fast RPM approach proposed by Ewert et al. [19]. The RPM method synthetizes a 2-D turbulent velocity slice from 3-D synthetic turbulence. According to the literature $[19,26]$ and numerical tests, considering 3-D synthetic turbulence leads to a better prediction of the spectrum frequency peak and of the spectral decay at high frequency compared to 2-D synthetic turbulence. For computational efficiency, the convolution product in Eq. (15) is evaluated as a series of 1-D filtering operations thanks to the separation of the Gaussian filter in the spatial directions. The RPM method is implemented in a Siemens in-house research code written in C++. Details about the implementation can be found in the literature [19] and only the main characteristics of the method are recalled here.

The RPM computational domain $\Omega_{0}$ is defined as a 2-D rectangular patch, as illustrated in Fig. 2. The stream function $\psi^{\prime}$ is evaluated at the nodes of a Cartesian mesh of constant grid spacing $h_{\mathrm{RPM}}$. For trailing-edge noise problems, it has been reported [26] that the use of $h_{\mathrm{RPM}}=0.0005 c$ yield satisfactory turbulence reconstructions, where $c$ is the length of the airfoil chord. The random field $W$ in Eq. (15) is discretized by a cloud of $N$ particles, shown in blue. The particles are initially randomly distributed in the domain $\Omega_{0}$ following a uniform distribution. The value of $N$ must be chosen sufficiently high to compute velocity fluctuations that statistically follow the prescribed turbulence properties [18]. In the literature, the number of particles per RPM grid cell $N_{\mathrm{ppc}}$ usually varies between 2.5 and $10[18,26,37,46]$.

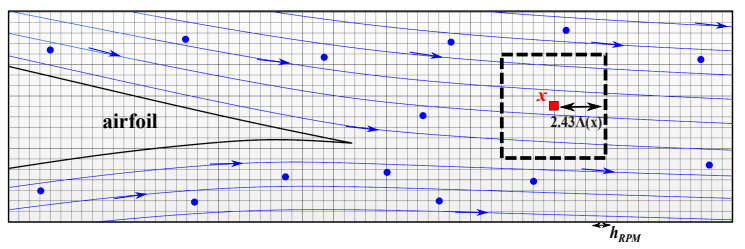

Fig. 2 2-D rectangular RPM domain near an airfoil: • particles, $\rightarrow$ mean velocity streamlines, $\square$ point $x$ where the RPM velocity is evaluated.

The particles are convected by the mean flow velocity $\boldsymbol{u}_{0}$ preliminarily computed on the Cartesian grid. When the values of $\boldsymbol{u}_{0}$ are prescribed from RANS, the velocity field at the RPM grid nodes is obtained from the CFD values using 
a bi-linear interpolation. The values of $\boldsymbol{u}_{0}$ at the particle locations are then bi-linearly interpolated from the velocity values at the RPM grid points. At each time iteration $\Delta t_{\mathrm{RPM}}$ of the RPM simulation, the positions of the particles are updated using a first-order Euler time-integration scheme. In order to keep the number of particles constant in the domain $\Omega_{0}$, the particles exiting the domain at the outflow boundary are re-injected at the inflow boundary of $\Omega_{0}$ [50]. The inflow and the outflow boundaries are defined by the orientation of the mean flow velocity vector.

In order to properly convect particles, the time step $\Delta t_{\mathrm{RPM}}$ is chosen so that the particles do not travel over distances larger than one RPM grid cell between two consecutive time iterations [25]:

$$
\Delta t_{\mathrm{RPM}} \leq \frac{h_{\mathrm{RPM}}}{\max \left(\left|\boldsymbol{u}_{0}(\boldsymbol{x})\right|\right)}
$$

In order to compute the stream function $\psi^{\prime}$ using Eq. (15), the mean flow values $\rho_{0}, k, \Lambda, \tau$ obtained analytically or from RANS are also initially prescribed at the nodes of the RPM grid. The RPM velocity fluctuations at the nodes of the Cartesian grid are computed from the values of $\psi^{\prime}$ using optimized high-order finite difference schemes on nine points [51]. Near a boundary, the size of the stencil of the centered spatial finite difference scheme is decreased from nine to three points. The vorticity fluctuations are then calculated from the velocity field $\boldsymbol{u}^{\prime}$ using a second-order centered finite-difference scheme. In practice, the high-order finite difference schemes [51] correctly resolve the wavelengths discretized by at least five points. The length scale $\Lambda$ has thus to verify the condition $\Lambda \geq 4 h_{\mathrm{RPM}}$. If it is not the case at one point (e.g in problems where the length scale $\Lambda$ includes very small values as typically found close to walls), as the grid spacing is constant and cannot be infinitely small due to prohibitive computational cost, the minimum value of the length scale is set to $\Lambda_{\min }=4 h_{\mathrm{RPM}}$.

The computational cost of the RPM method is mainly driven by the filtering operation in Eq. (15). To reduce this cost, the integral in Eq. (15) is not computed over the whole domain $\Omega_{0}$. It is assumed that a particle is no longer contributing to the stream function field when it is located at a distance larger than $2.43 \Lambda(\boldsymbol{x})$ from the point where it is evaluated. For the Gaussian filter in Eq. (16), a cut-off value of $2.43 \Lambda(\boldsymbol{x})$ is selected in this work. It corresponds to a truncation of the exponential function when its value is less than $\epsilon=10^{-4}$. The use of values of $\epsilon$ between $10^{-2}$ and $10^{-4}$ is recommended $[13,21,52]$.

\section{Benefits of a frequency-domain approach to simulate sound propagation}

When the RPM method is coupled with an acoustic propagation solver, the sampling time $\Delta t_{\mathrm{ac}}$ at which the RPM sources are recorded for the acoustic simulation can differ from the RPM time step $\Delta t_{\mathrm{RPM}}$. The value of $\Delta t_{\mathrm{ac}}$ is usually determined as a function of the maximum frequency $f_{\max }$ of interest to compute the sound level spectra. Following the 
Nyquist sampling criterion, the time step $\Delta t_{\mathrm{ac}}$ must verify the condition:

$$
\Delta t_{\mathrm{ac}} \leq 1 /\left(2 f_{\max }\right) .
$$

This condition has to be satisfied in both time-domain and frequency-domain propagation approaches. However, when the RPM method is coupled with a time-domain propagation solver using an explicit time discretization scheme, besides the condition (21), the time step $\Delta t_{\mathrm{ac}}$ must be chosen in order to verify the CFL restriction [28]. In the absence of mean flow, the CFL restriction can be written as:

$$
\Delta t_{\mathrm{ac}} \leq v^{*} \frac{h_{\mathrm{ac}}}{c_{0}} \quad \text { with } \quad v^{*}>0
$$

where $h_{\mathrm{ac}}$ is the size of a local cell in the acoustic mesh and $v^{*}$ is the maximum CFL number to guarantee the stability of the time discretization scheme. In practice, the presence of very small grid cells is often needed to accurately represent geometries. This increases the computational cost, especially for broadband noise applications where a long time period has to be simulated in order to predict the acoustic levels at low frequencies.

Coupling the RPM method with a frequency-domain propagation solver has the advantage of avoiding the CFL restriction (22). However, in the frequency-domain approach, the RPM and the sound propagation simulations cannot be done synchronously, as in the time-domain approach. More precisely, the sound sources are first computed in the time domain by RPM. They are stored and then converted into the frequency domain at the end of the RPM simulation before performing the sound propagation calculation.

\section{Numerical verification of the RPM-Helmholtz approach}

\section{A. Problem definition}

In order to verify the performance of the RPM-Helmholtz approach presented in this study, a scalar source $q_{c}^{\prime}$, stochastically generated using the RPM method, is introduced in a medium at rest [31]. In this problem, all the quantities are normalized by the density $\rho_{0}$, the speed of sound $c_{0}$ and the characteristic size of the RPM source region denoted $\ell$. The RPM source region is defined by the two-dimensional rectangular domain $\Omega_{0}$ shown in Fig. 3. The domain $\Omega_{0}$ extends from $x=-\ell$ to $x=\ell$ and from $y=-0.5 \ell$ to $y=0.5 \ell$.

The RPM source term $q_{c}^{\prime}$ computed in $\Omega_{0}$ is defined by the space-time correlation:

$$
\left\langle q_{c}^{\prime}(\boldsymbol{x}, t) q_{c}^{\prime}(\boldsymbol{x}+\boldsymbol{r}, t+s)\right\rangle=R_{g}(\boldsymbol{x}) \exp \left(-\frac{s}{\tau}-\frac{\pi\left|\boldsymbol{r}-\boldsymbol{u}_{0} s\right|^{2}}{4 \Lambda^{2}}\right)
$$

where $\boldsymbol{x}=(x, y), \boldsymbol{u}_{0}=\left(u_{0}, 0,0\right)$ is the convection velocity in the source region with $u_{0}=0.3 c_{0}, \Lambda=0.05 \ell$ is the target 


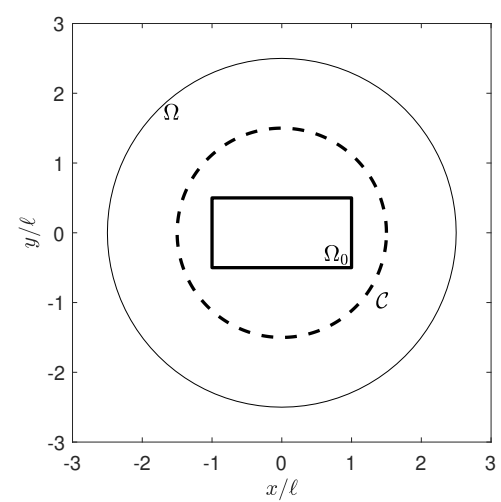

Fig. 3 Representation of the computational domain considered for the Gaussian source case: RPM domain $\mathbf{\Omega}_{\mathbf{0}}$ and FEM domain $\Omega$ with circle $C$ where the sound levels are computed.

correlation length scale, $\tau=0.67 \ell / c_{0}$ is the target correlation time scale, and $R_{g}(\boldsymbol{x})$ is the variance of the fluctuating source defined by the Gaussian function:

$$
R_{g}(\boldsymbol{x})=\left\langle q_{c}^{\prime}(\boldsymbol{x})^{2}\right\rangle=\exp \left(-\frac{x^{2}}{2 b_{1}{ }^{2}}-\frac{y^{2}}{2 b_{2}^{2}}\right)
$$

where $b_{1}=0.2 \ell$ and $b_{2}=0.1 \ell$ are the Gaussian half-widths. Note that following reference [31], a non-zero convection velocity is considered in the source model, but the sound propagation is carried out in a medium at rest. Equation (8), with $\widehat{\boldsymbol{q}}_{m}=\mathbf{0}$, is solved using the FEM approach presented in section II.A.3. The pressure field $\hat{p}$ radiated from the source is recorded on a circle $C$ of radius of $1.5 \ell$ centered on the origin $(0,0)$. The Sound Pressure Levels (SPL) are then computed from the values of $|\hat{p}|^{2}$. At point $\boldsymbol{x}^{\prime}$ and at frequency $f$, it yields:

$$
\operatorname{SPL}\left(\boldsymbol{x}^{\prime}, f\right)=10 \log _{10}\left(\frac{\left|\hat{p}\left(\boldsymbol{x}^{\prime}, f\right)\right|^{2}}{p_{\mathrm{ref}}^{2}}\right)
$$

where $p_{\text {ref }}=2 \times 10^{-5} \mathrm{~Pa}$ is the reference pressure. The sound levels are compared with the analytical solution derived in reference [31]. The analytical expression of the two-sided sound spectrum at a point $\boldsymbol{x}^{\prime}$ can be written as a function of the angular frequency as follows:

$$
\mathcal{S}\left(\boldsymbol{x}^{\prime}, \omega\right)=\int_{\Omega_{0}} 8 R_{g}(\boldsymbol{x}) \tau \Lambda^{2}\left|\widetilde{G}\left(\boldsymbol{x}, \boldsymbol{x}^{\prime}, k_{\omega}\right)\right|^{2} \frac{\exp \left[-\frac{\omega^{2} \Lambda^{2}}{\pi c_{0}^{2}}\right]}{1+\omega^{2} \tau^{2}\left(1-\frac{u_{0}}{c_{0}} \cos \theta\right)^{2}} \mathrm{~d} \boldsymbol{x}
$$

where $\theta$ is the angle between $O \boldsymbol{x}^{\prime}$ and the $x$-axis, and $\widetilde{G}$ is the Green's function associated to the wave operator in Eq. (8) (assuming the convention $\exp (+\mathrm{i} \omega t))$ scaled by a factor $k_{\omega} /\left(\mathrm{i} c_{0}\right)$ :

$$
\widetilde{\mathrm{G}}\left(\boldsymbol{x}, \boldsymbol{x}^{\prime}, k_{\omega}\right)=\frac{k_{\omega}}{4 c_{0}} H_{0}^{2}\left(k_{\omega}\left|\boldsymbol{x}-\boldsymbol{x}^{\prime}\right|\right) .
$$


where $H_{0}^{2}$ is the zero-order Hankel function. In order to assess the accuracy of the FEM propagation technique, the sound levels are also computed using the free-field Green's function $\widetilde{G}$ instead of FEM to solve the 2-D Helmholtz equation (8). In this case, the values of the RPM source term $\widehat{q}_{c}$ on the RPM grid are used, and no interpolation on an acoustic mesh is needed. The solution obtained from the free-field Green's function is denoted RPM-GreenFunction in the following.

\section{B. Numerical set-up}

For the RPM simulation, a Cartesian mesh with a constant grid spacing $h_{\mathrm{RPM}}=\Lambda / 5=0.01 \ell$ is used. The simulation is carried out over a time period of $T=3200 \ell / c_{0}$ with a time step $\Delta t_{\mathrm{RPM}}=\Delta t_{\mathrm{ac}}=0.01 \ell / c_{0}$. In order to set the number of particles in RPM domain, simulations have been done using numbers of particles per cell $N_{\mathrm{ppc}}$ of 1, 2.5 and 5. For this test case, increasing the value of $N_{\text {ppc }}$ has a negligible effect on the acoustic results. For the sake of conciseness, only the results obtained using $N_{\mathrm{ppc}}=1$ are presented, yielding $2 \times 10^{4}$ particles in the RPM domain.

For the sound propagation, a circular computational domain of radius of $2.5 \ell$ centered on the origin is defined (see Fig. 3). This domain is surrounded by a ten-layer PML region to absorb the outgoing waves [39]. An unstructured triangular mesh characterized by a constant element size $h_{\mathrm{ac}}=0.1 \ell$ is designed using the open source mesh generator Gmsh [53]. To obtain statistically converged spectra, as described in section II.B.2, the RPM time signal computed over a period $T$ is divided into $N_{\text {seg }}=319$ segments of length $T_{\text {seg }}=20 \ell / c_{0}$ which overlap by $50 \%$, yielding a frequency resolution of $\Delta f=1 / T_{\mathrm{seg}}=0.05 c_{0} / \ell$. The convergence of the acoustic results with respect to the number of segments $N_{\text {seg}}$, presented in Appendix VI.A, shows that good trends are obtained even when using only 19 time segments. The FEM simulation is then carried out for each time segment and each frequency within the range $\left[0.05 c_{0} / \ell-10 c_{0} / \ell\right]$. The simulations are performed using a target accuracy $E_{T}$ of $1 \%$, leading to polynomial orders $p_{\text {FEM }}$ varying from 2 to 7.

\section{Results}

The SPL computed at a distance of $r=\sqrt{x^{2}+y^{2}}=1.5 \ell$ from the origin for angles $\theta=0^{\circ}$ and $\theta=90^{\circ}$ are presented in Fig. 4 as a function of the normalized frequency $f \ell / c_{0}$. The results obtained from the RPM-FEM and the RPM-GreenFunction approaches are compared with the analytical solution. The spectra obtained from the RPM-FEM approach show an excellent agreement with the RPM-GreenFunction spectra. This demonstrates that the FEM solver accurately propagates sound waves and that the sound sources computed from RPM are correctly interpolated on the FEM mesh. The amplitude of the spectra obtained from RPM is in good agreement with the analytical solution over the frequency range $0.8 c_{0} / \ell \leq f \leq 10 c_{0} / \ell$. For $f<0.8 c_{0} / \ell$, the differences between the spectra are attributed to the RPM simulation time $T$ which is too short to ensure a good convergence of the spectra in time.

In order to examine the quality of the RPM-FEM results at different angles $\theta$, the SPL directivity at $r=1.5 \ell$ 
(a)

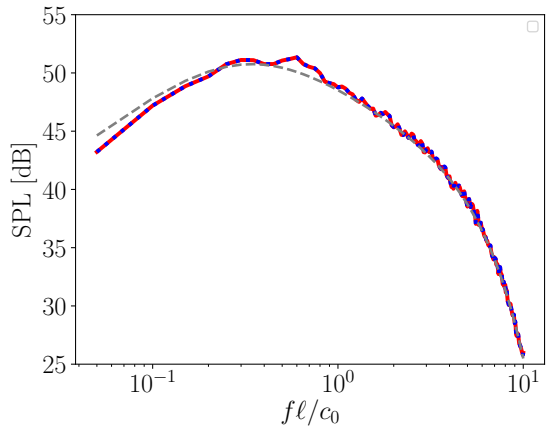

(b)

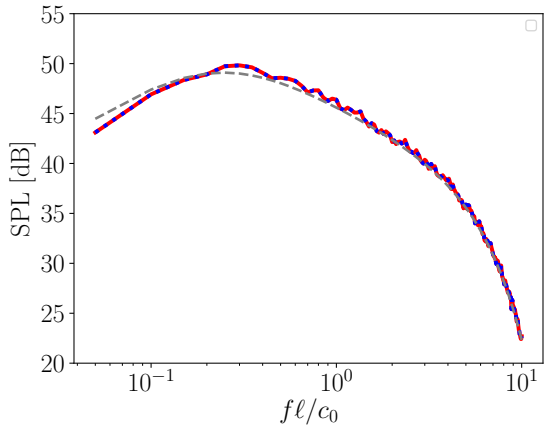

Fig. 4 SPL at $r=1.5 \ell$ for angles of a) $\theta=0^{\circ}$ and b) $\theta=90^{\circ}$ : — RPM-FEM and $\cdots$ RPM-GreenFunction with $N_{\text {seg }}=319$, and - - analytical solution.

from the origin is presented in Fig. 5 for frequencies of $2.5 c_{0} / \ell, 5 c_{0} / \ell, 7.5 c_{0} / \ell$ and $10 c_{0} / \ell$. The RPM-FEM and the RPM-Greenfunction results are compared with the analytical solution. For all the frequencies, the RPM-FEM and RPM-GreenFunction directivities are in excellent agreement, as previously reported in Fig. 4. Overall, their shape and amplitude follow the analytical solution.

(a) $f=2.5 c_{0} / \ell$

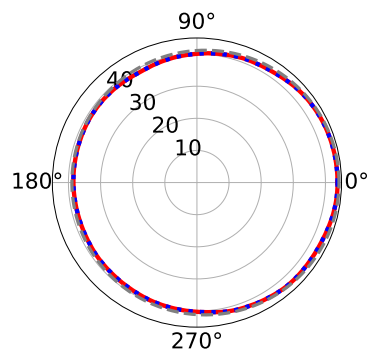

(b) $f=5 c_{0} / \ell$

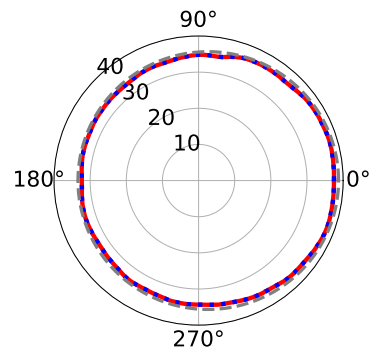

(c) $f=7.5 c_{0} / \ell$

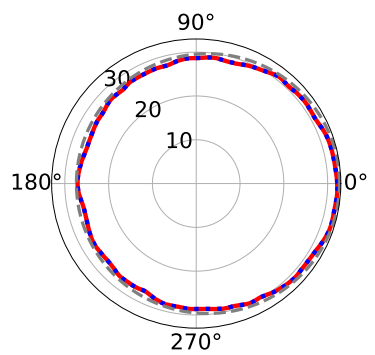

(d) $f=10 c_{0} / \ell$

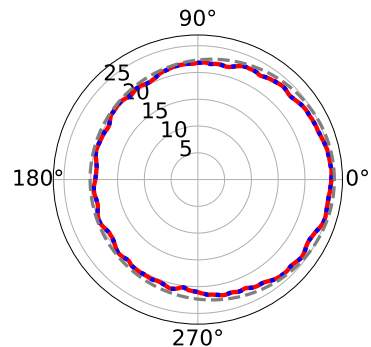

Fig. 5 Directivities of the SPL at $R=1.5 \ell$ obtained for $N_{\text {seg }}=319$ : - RPM-FEM, $\cdots$ RPM-GreenFunction and --- analytical solution.

\section{Application to a NACA0012 airfoil}

In order to examine the validity of the Helmholtz-based approach in the presence of a mean flow, the sound radiated by the trailing-edge of a NACA0012 airfoil at zero angle of attack is computed for flows at Mach numbers $M$ of 0.1118 and 0.1664. Both APE and Helmholtz FEM simulations are carried out. These NACA0012 configurations correspond to cases 1 and 4 of BANC-II workshop [54], for which experimental data are available. The airfoil has a chord length $c$ of $0.4 \mathrm{~m}$ and a span $L$ of $1 \mathrm{~m}$. The flow configuration at $M=0.1118$ is characterized by a free-stream velocity of $u_{\infty}=37.7 \mathrm{~m} / \mathrm{s}$, a speed of sound of $c_{0}=337 \mathrm{~m} / \mathrm{s}$ and a chord-based Reynolds number of $R e_{c}=1 \times 10^{6}$. At $M=0.1664$, the flow properties are $u_{\infty}=56 \mathrm{~m} / \mathrm{s}, c_{0}=336.3 \mathrm{~m} / \mathrm{s}$ and $R e_{c}=1.5 \times 10^{6}$. 


\section{A. RANS simulations}

In order to compute the flow statistics $\boldsymbol{u}_{0}, k$, and $\Lambda$ required to compute the sound sources, 2-D RANS simulations are performed using commercial CFD software Simcenter STAR-CCM+. The simulations are performed using the SST $k-\omega$ turbulence model [47], with second-order accurate solution for all variables. The computational domain has a size of $40 c$ in each direction, with the trailing-edge of the airfoil located at $x=y=0$. At the inlet of the domain, the free-stream velocity $u_{\infty}$ and a low turbulent intensity [54] of $0.001 \%$ are imposed. At the outlet of the domain, the ambient pressure $p_{0}=\rho_{0} c_{0}^{2} / \gamma$ is set with $\gamma=1.4$ the heat capacity ratio. A no-slip boundary condition is used at the airfoil surface. A C-type structured mesh of about 210,000 cells is designed, with a grid spacing in the wall-normal direction satisfying $y+<0.6$ to ensure a good spatial resolution of the boundary layer. No tripping is applied to trigger the laminar-turbulent transition in the boundary layer. The turbulent integral length scale $\Lambda$ is computed from the turbulent kinetic energy $k$ and the specific dissipation rate $\omega_{t}$ obtained from RANS using Eq. (19) with $l_{\text {factor }}=6$. The RANS results in the near-wake at $x=0.0038 c$, including the mean axial velocity, the turbulent kinetic energy $k$ and the length scale $\Lambda$ profiles, are compared with experimental data [54] in Fig. 6. Overall, the velocity and energy profiles show a fair agreement with the experimental data, except for $y<0.004 \mathrm{~m}$ where the values of $k$ are underpredicted. The length scale computed from CFD is smaller than the measured values, possibly due to an overprediction of the dissipation $\omega_{t}$ in RANS. Similar RANS results are reported in the literature [26, 54].

(a)

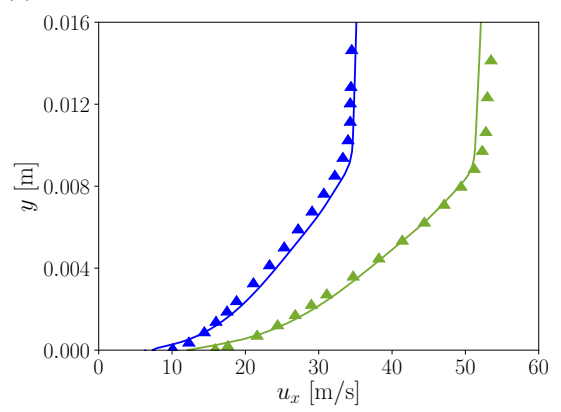

(b)

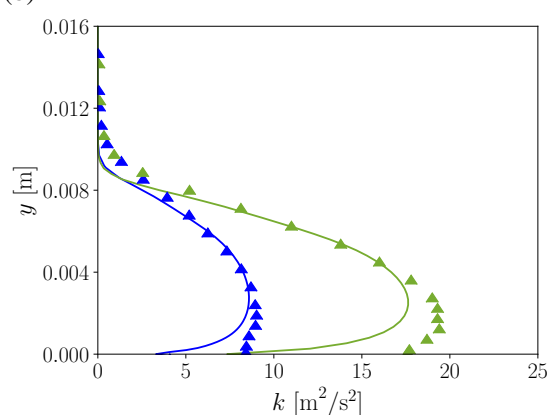

(c)

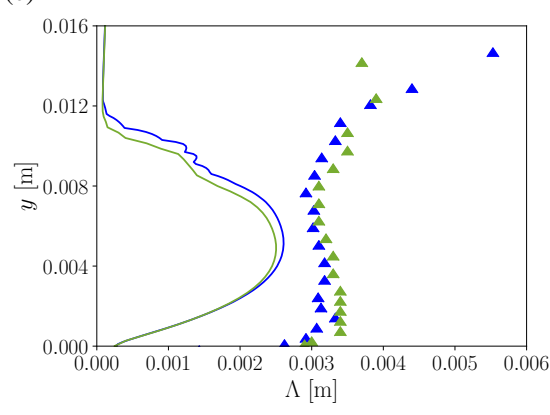

Fig. 6 Representation of (a) the mean axial velocity, (b) the turbulent kinetic energy and (c) the turbulent length scale at $x_{c}=0.0038 c$ : RANS at $-M=0.1118$ and $-M=0.1664$, IAG experiment [54] at $\triangle M=0.1118$ and $\triangle M=0.1664$.

\section{B. Calculation of the sound sources using RPM and sound propagation using FEM}

\section{Set-up of RPM simulations}

The sound sources, obtained from the RPM method, are computed following the best practices recommended in the literature $[26,55]$. As the inclusion of a time-decorrelation of turbulence in the source model is found to have a negligible effect on the radiated sound field for edge noise problems [13,56], all the RPM simulations are performed assuming frozen turbulence. 
The extent of the RPM domain is chosen to include the boundary layer profile and the high turbulent kinetic energy levels near the airfoil trailing edge. Following the recommendations of Rautmann for trailing-edge noise problems [55], a RPM domain $\Omega_{0}$ of size $L_{x}=0.4 c$ and $L_{y}=0.1 c$ centered at the trailing edge is chosen, with a grid resolution of $h_{\mathrm{RPM}}=5 \times 10^{-4} c$. The number of particles per RPM cell is equal to $N_{\mathrm{ppc}}=2.5$ [55], and a Gaussian filter threshold $\epsilon$ of $10^{-4}$ is used [13].

The noise predictions obtained experimentally are reported in third-octave bands for $f=1 \mathrm{kHz}$ up to $10 \mathrm{kHz}$. The frequency range $[560-11120] \mathrm{Hz}$ is considered for the numerical simulations. The RPM simulations are carried out over a time period $T$ of $0.392 \mathrm{~s}$ with a time step $\Delta t_{\mathrm{RPM}}=1.96 \times 10^{-6} \mathrm{~s}$ chosen to satisfy Eq. (22). This set-up leads to statistically converged turbulence statistics near the airfoil trailing edge. In order to compute the sound sources, the RPM data are then sampled every 15 time steps $\left(\Delta t_{\mathrm{ac}}=2.94 \times 10^{-5} \mathrm{~s}\right)$, corresponding to a Nyquist frequency of $f=17 \mathrm{kHz}>10 \mathrm{kHz}$ to avoid aliasing. In order to prevent the generation of spurious noise due to an abrupt truncation of the source field at the boundaries of the RPM domain $\Omega_{0}$, the values of $L^{\prime}$ are smoothly damped to zero near the boundaries of $\Omega_{0}$. For this purpose, a Hann windowing function $w$ is applied in $x$ and $y$ directions. In direction $s$, it is defined as:

$$
w(x)=\frac{1}{2}\left[1+\cos \left(\frac{\pi}{x_{\mathrm{b}}}\left(x-x_{0}\right)\right)\right]
$$

where $x_{0}$ is the position where the filter starts to act and $x_{\mathrm{b}}$ is the width of the damping region. In direction $x$, the windowing is applied over a length of $x_{\mathrm{b}}=0.25 L_{x}$ from the left and right boundaries. In the direction $y$, the same function $w$ is applied with a windowing length of $y_{\mathrm{b}}=0.2 L_{y}$ at the top and bottom boundaries.

\section{Set-up of FEM simulations}

A 2-D circular computational domain of radius $1.25 c$ centered on the trailing edge of the airfoil is considered. At its external boundary, a PML region, made of four layers, is used in order to absorb the outgoing waves [39]. An unstructured acoustic mesh is designed using Gmsh [53]. It is composed of 6-noded triangular elements, except in the PML region where 9-noded quadrangular elements are used. At the airfoil walls, the meshes are refined with quadratic elements of size $0.004 c$ in order to accurately represent the geometry. In the vicinity of the trailing edge where the RPM sound sources are computed, the meshes are made of elements of size $h_{\mathrm{ac}}$ source. It is often pointed out in the literature that the acoustic mesh in the source region must be as fine as the RPM grid [55]. For the NACA0012 cases, a resolution of $h_{\mathrm{ac} \text { source }}=4 h_{\mathrm{RPM}}$ in the source region is found sufficient to ensure mesh independent results. The largest elements are located at the PML interface, and have a characteristic size of $h_{\mathrm{ac} \max }=0.083 c$ chosen so that $p_{\mathrm{FEM}} \leq 10$.

In the Helmholtz simulations, uniform mean flow density $\rho_{0}$ and speed of sound $c_{0}$ are prescribed. In the APE simulations, the mean flow obtained from RANS is interpolated at the integration points of the acoustic mesh using a linear scattered interpolation approach [57] available in Matlab. 
The sound source term $\boldsymbol{L}^{\prime}$, calculated in the time domain on the RPM Cartesian grid, is converted to the frequency domain and then interpolated on the acoustic mesh, as described in section II.B.2. For this purpose, the RPM time signal $L^{\prime}$ is divided into $N_{\text {seg }}=30$ segments of period $T_{\text {seg }}=0.025 \mathrm{~s}$ which overlap by $50 \%$, resulting in a frequency resolution of $\Delta f=1 / T_{\text {seg }}=40 \mathrm{~Hz}$. The FEM simulations are performed for the 30 right-hand sides over the frequency range [560 - 11240] Hz with the frequency resolution $\Delta f$. Resorting to the a priori error indicator described in [43], the polynomial order distribution is determined at each frequency prior to the calculation so as to reach a user-defined target accuracy $E_{T}=0.5 \%$. Thanks to this high-order adaptive FEM approach, a single input mesh can be used to efficiently cover the whole frequency range. This FEM order distribution is discussed in more details in section V.

The sound levels in the far-field region are computed at a distance of $r_{\mathrm{obs}}=1 \mathrm{~m}$ from the trailing-edge. In the Helmholtz simulation, the values of $\hat{p}$ in the far-field region are determined from the pressure $\hat{p}$ and its normal derivative recorded at the PML interface using a 2-D Kirchhoff extrapolation method [58]. In the APE-4 simulation, the far-field solution is extrapolated from the values of $\hat{\rho}, \hat{\boldsymbol{u}}$ and $\hat{p}$ at the PML interface using a 2-D frequency-domain convective Ffowcs Williams and Hawkings $(\mathrm{FW}-\mathrm{H})$ method [59]. The power spectral density $S_{p p}$ is then computed from the values of $|\hat{p}|^{2}$ averaged over the 30 segments. In order to determine 3-D sound predictions from the 2-D simulations, the SPL at location $\boldsymbol{x}=\left(r_{\mathrm{obs}}, \theta_{\mathrm{obs}}\right)$ is computed from the following relation:

$$
\operatorname{SPL}_{3 \mathrm{D}}(\boldsymbol{x}, \omega)=10 \log _{10}\left(\frac{S_{p p}(\boldsymbol{x}, \omega) \Delta f}{p_{\text {ref }}^{2}}\right)+10 \log _{10}\left(\frac{1.4}{2 \pi} \frac{L}{r_{\mathrm{obs}}} M\right)
$$

where the second term in Eq. (29) is a 2-D to 3-D correction term [19]. A constant offset of -12 dB has been applied to all the acoustic spectra presented in this section in order to match the the experimental levels. To the authors' knowledge, similar calibration procedures are systematically reported in the literature when using RPM for trailing-edge noise problems $[19,22,26]$.

\section{Results}

The turbulent kinetic energy profiles obtained from RPM and RANS at $x=0$ are compared in Figs. 7(a) and 7(b). Similar results are obtained for the two flow configurations with energy peaks smoother in RPM than in RANS. These discrepancies are attributed to the spatially varying turbulence properties and to the choice to compute the amplitude $A$ of the RPM filter at the particle positions $\boldsymbol{x}^{\prime}$ leading to an effective source variance that does not accurately follow the target value prescribed from RANS [20]. The sound pressure levels obtained numerically and experimentally at $1 \mathrm{~m}$ and $270^{\circ}$ below the trailing-edge are represented in third-octave bands in Fig 7(c), as a function of the center frequency $f_{c}$. The shape of the RPM-FEM spectra shows a reasonable agreement with the experimental data for $f_{\mathrm{c}} \leq 4 \mathrm{kHz}$. At higher frequencies, the RPM-FEM spectra display a sharper decrease most likely due to the use of a Gaussian energy spectrum to model the turbulence in the RPM method [26]. The Helmholtz and APE results agree well $f_{\mathrm{c}} \leq 4 \mathrm{kHz}$. 
Differences of 2-3 dB are reported for $f_{\mathrm{c}}>4 \mathrm{kHz}$.

The far-field directivities computed from the Helmholtz and APE approaches for three Helmholtz numbers $k_{\omega} c=5$, 10 and 20 (corresponding to $f=680,1360$ and $2680 \mathrm{~Hz}$ ) are shown in linear scale in Fig. 8. The directivity patterns are similar to those reported by Cozza et al. in reference [21]. Only slight differences are reported between the Helmholtz and APE-based solutions. These results suggest that Helmholtz solver can be used at least up to $M=0.16$.

(a)

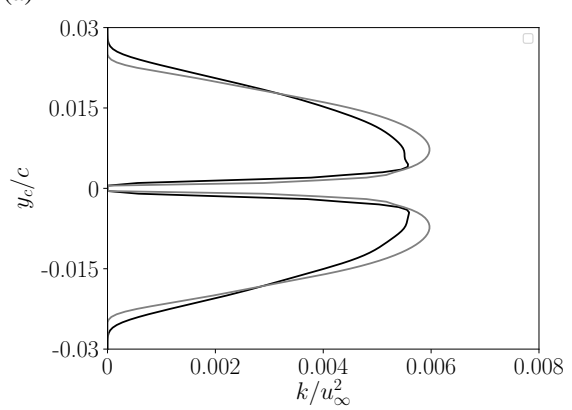

(b)

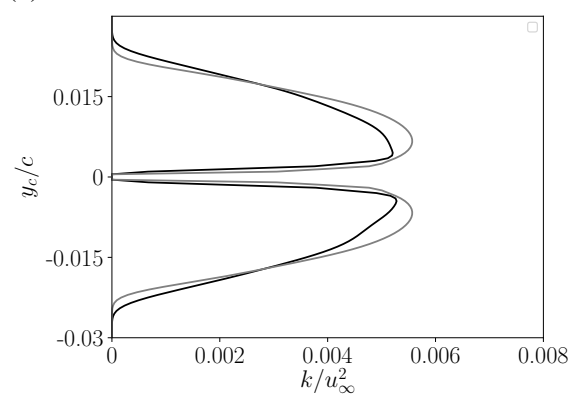

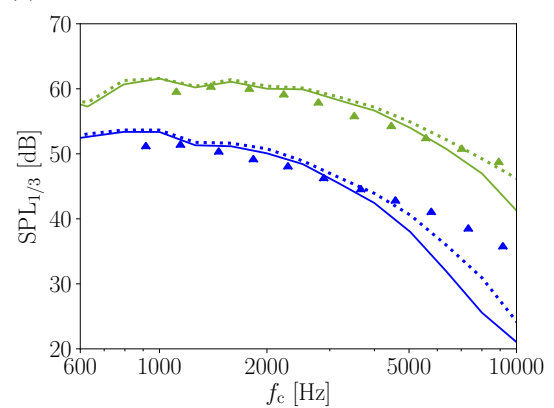

Fig. 7 Representation of the turbulent kinetic energy at $x=0$ for configurations (a) \#1 and (b) \#2: — RPM and - RANS and (c) $1 / 3$ octave band spectra at $r_{\mathrm{obs}}=1 \mathrm{~m}$ and $\theta_{\mathrm{obs}}=270^{\circ}$ : Helmholtz solver at - $M=0.1118$ and $-M=0.1664$, and APE-4 solver at $\cdots M=0.1118$ and $\cdots M=0.1664$, and $\triangle \triangle$ DLR experiments [54].

(a) $M=0.1118-k_{\omega} c=5$

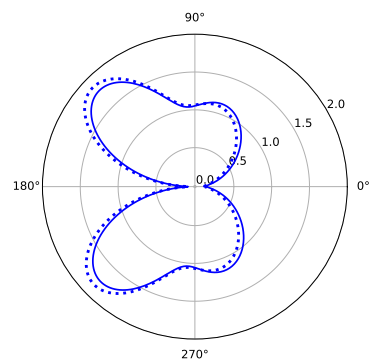

(d) $M=0.1664-k_{\omega} c=5$

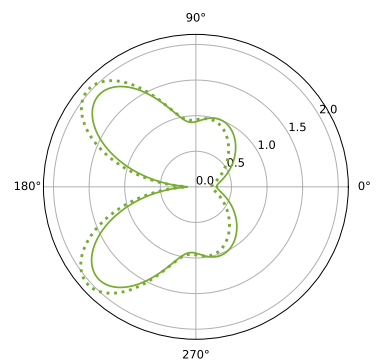

(b) $M=0.1118-k_{\omega} c=10$

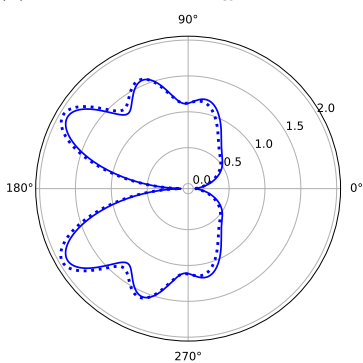

(e) $M=0.1664-k_{\omega} c=10$

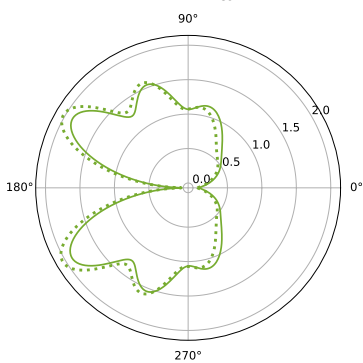

(c) $M=0.1118-k_{\omega} c=20$

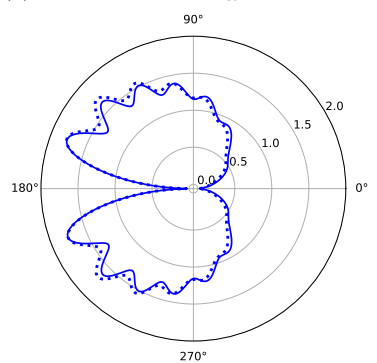

(f) $M=0.1664-k_{\omega} c=20$

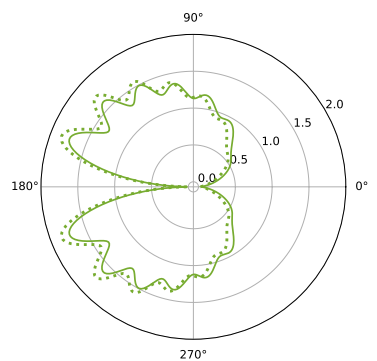

Fig. 8 Directivities at $r_{\text {obs }}=1 \mathrm{~m}$ : Helmholtz solver at $-M=0.1118$ and $-M=0.1664$, and APE-4 solver at $\cdots M=0.1118$ and $\cdots M=0.1664$.

\section{Application to low Mach number trailing edge noise prediction}

The numerical methodology described in section II is now applied to predict the noise radiated from the trailing-edge of a Controlled-Diffusion (CD) airfoil for a flow Mach number of $M=0.047$. The objective is to examine if the 
conclusions obtained for the NACA0012 airfoil in section IV still hold for a different airfoil geometry and a different Mach number. The CD airfoil, displayed in Fig 9(b), is selected as it has been thoroughly investigated experimentally [32, 33] and numerically $[3,34]$ in the literature. Characterized by a curved shape, it has a chord length $c$ of $0.1356 \mathrm{~m}$ and a span $L$ of $0.3 \mathrm{~m}$. Its cross-section in the spanwise direction is constant, which allows us to perform a 2-D numerical simulation and to retrieve the 3-D radiated sound field using a 2-D to 3-D correction [19]. The CD airfoil coordinates are provided as supplemental material. The airfoil is simulated at an angle of attack $\alpha$ of $8^{\circ}$. The flow around the airfoil is characterized by a free-stream velocity of $u_{\infty}=16 \mathrm{~m} / \mathrm{s}$ and a Reynolds number based on the chord length of $R e_{\mathrm{c}}=1.6 \times 10^{5}$. The ambient density and the sound speed are equal to $\rho_{0}=1.225 \mathrm{~kg} / \mathrm{m}^{3}$ and $c_{0}=340 \mathrm{~m} / \mathrm{s}$.

\section{A. RANS simulation}

An incompressible 2-D RANS simulation is performed using commercial CFD software Simcenter STAR-CCM+. The computational domain is presented in Fig. 9(a). It is characterized by a length of $4.3 c$ in direction $x$ and a length of $2.5 c$ in direction $y$, with the trailing-edge of the airfoil located at $x=y=0$. The flow is oriented from the left to the right. This domain size is too small for a typical airfoil calculation, in general, but the results, in this case, are verified a posteriori by comparisons with existing RANS results found in literature [34]. In the experiment [33], the airfoil is installed in an anechoic open-jet wind tunnel and the jet deflection strongly influences the aerodynamic loading applied to the airfoil [60]. In order to account for these effects, the mean velocity profile imposed at the inlet boundary is extracted from a RANS computation done by Christophe [34] in a larger domain including the complete open-jet wind tunnel configuration with the nozzle. This inlet velocity profile is attached as supplemental material. The turbulent intensity at the inlet of the domain is set to $0.7 \%$, corresponding to the value measured in the experiment [33]. As in section IV, a no-slip boundary condition for the velocity is enforced at the airfoil wall and the ambient pressure $p_{0}$ is imposed at the domain outlet. The SST $k-\omega$ turbulence model [47] is chosen as it provides results in good agreement with the experimental data, as reported in [60].

The RANS simulation is carried out using a structured mesh of about 159,000 cells. A representation of the mesh near the airfoil is given in Fig. 9(b). It is refined in the vicinity of the leading edge and the trailing edge. At the airfoil wall, the grid spacing in the wall-normal direction is such that $y^{+}<0.85$ in order to ensure a good spatial resolution of the boundary layer. No tripping is applied to trigger the laminar-turbulent transition in the boundary layer. The influence of the grid resolution on the RANS results has been examined and only converged results are presented here for brevity. 
(a)

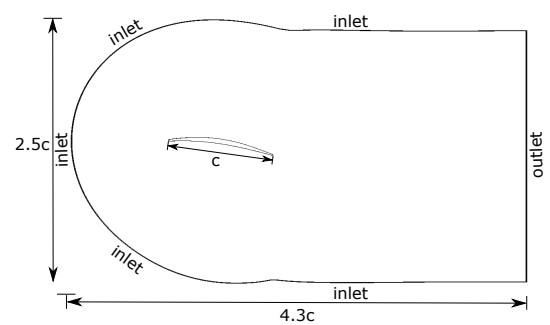

(b)

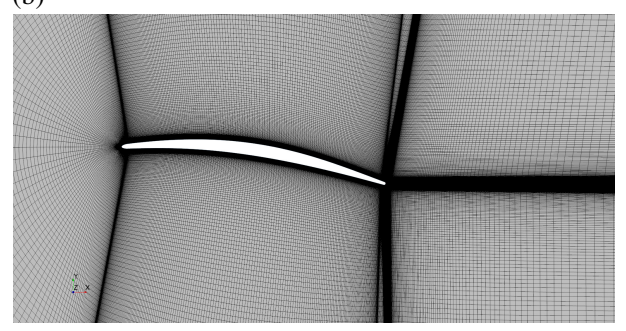

Fig. 9 a) RANS computational domain and b) mesh close to the airfoil.

The results of the RANS simulation are compared with the RANS results of Christophe [34] and the experimental data of Moreau et al. $[32,33]$. The distribution of the pressure coefficient $C_{p}$ along the airfoil surface is presented in Fig. 10(a). The values of $C_{p}$ are plotted with respect to the chordwise coordinate $x_{c}=x / \cos \alpha$ used to represent data along the chord of the airfoil, with $x_{c}=0$ at the trailing-edge. A good agreement with numerical and experimental data of the literature is reported on the pressure side of the airfoil and on the suction side for $x_{c} \geq-0.85 c$. For $x_{c}<-0.85 c$ on the suction side, the $C_{p}$ profile obtained in this study presents slight differences with the RANS profile of [34], and more pronounced discrepancies with the experimental data. In this region, the transition is triggered by a flow separation resulting into a recirculation bubble $[34,60]$ which is shown on the axial velocity iso-contours plotted in Fig. 11. Note that the present RANS model is assuming the resolution of fully turbulent flows and is then not adapted to transitional phenomena as the ones observed in the leading-edge region.

In order to estimate the size of the bubble, the distribution of the skin friction coefficient $C_{f}$ along the airfoil suction side is represented in Fig. 10(b), as a function of $x_{c} / c$. The laminar separation area at the leading edge is identified as the region of negative skin friction coefficient. The streamwise extent of the recirculation bubble is of approximately $5.3 \% \mathrm{c}$ for the present RANS computation and of 3.1\%c for the RANS computation of Christophe [34]. In the present work, as the flow reattachment occurs further downstream, lower values of $C_{f}$ are obtained. Contrary to the $C_{p}$ profile, the prediction of the size of the bubble has a non-negligible influence on the $C_{f}$ profile near the leading edge. However, near the trailing edge, i.e. the region of interest in this study, the profile of the skin friction coefficient is similar to that of [34], slowly decreasing along the coordinate $x_{c}$.

The mean velocity $u_{t}$ in the tangential direction to the wall at $x=-0.14 c$ and $x=-0.02 c$ on the suction side of the airfoil is presented in Figs. 12(a) and 12(b), as a function of the normalized wall-distance $y_{\mathrm{w}} / c$. The boundary layer thickness increases as the distance from the trailing-edge decreases, as expected. The present results agree well with the RANS profiles [34] but under-predict the mean velocity compared to the experiment for $y_{\mathrm{w}}<0.03 c$. The boundary layer obtained numerically is thicker than the experimental one. This might be related to the mismatch of the pressure distribution at the leading-edge of the airfoil.

The turbulence intensity $\mathrm{TI}=\sqrt{2 k / 3 u_{\infty}^{2}}$ at $x=-0.14 c$ and $x=-0.02 c$ in the boundary layer on the suction side 
(a)

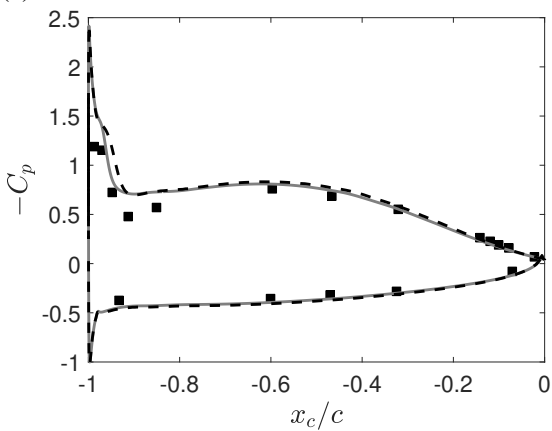

(b)

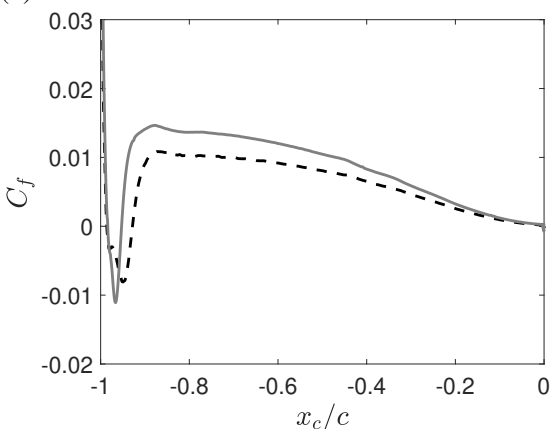

Fig. 10 Representation of a) the pressure coefficient $C_{p}$ along the airfoil wall and b) the skin friction coefficient $C_{f}$ along the airfoil suction side: - - - present RANS, — RANS Christophe [34] and $\square$ experiment [32, 33].

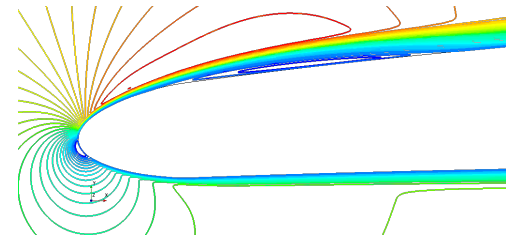

Fig. 11 Representation of the axial velocity iso-contours in the vicinity of the airfoil leading edge. The color scale is from $-0.1 u_{\infty}$ to $1.5 u_{\infty}$ from blue to red.

of the airfoil is plotted as a function of the normalized wall-distance $y_{\mathrm{w}} / c$ in Figs. 12(c) and 12(d). While a good match with the RANS results of Christophe [34] is reported, large discrepancies with the experiment are visible. At $x=-0.14 c$, for $y_{w}<0.25 c$, the turbulence intensity levels predicted numerically are underestimated with respect to the experimental data. In particular, the amplitude of the TI peak is under-predicted by $40 \%$ in RANS. The agreement with experimental data is slightly better at $x=-0.02 c$, with a peak amplitude under-estimated by about $25 \%$ in RANS. As suggested by Christophe [34], these differences might be due to difficulties in capturing the large scales emanating from the recirculation zone with the current RANS modelling.

(a)

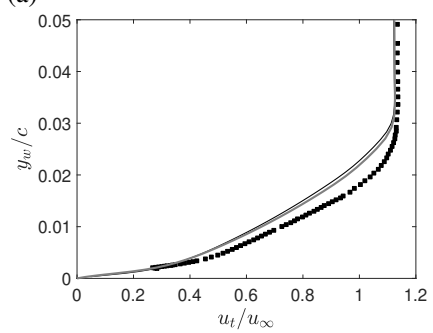

(b)

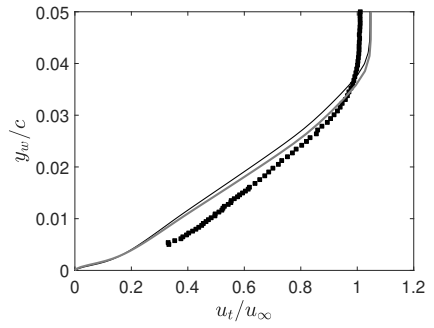

(c)

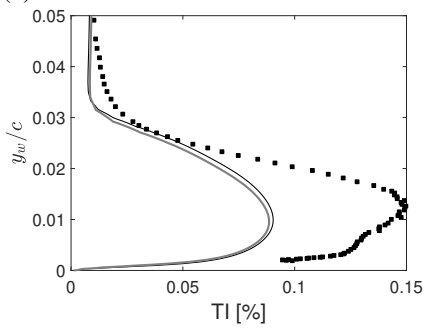

(d)

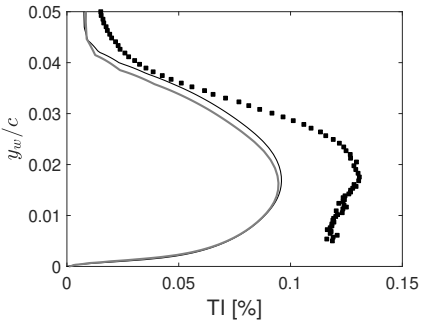

Fig. 12 Representation of the mean velocity $u_{t}$ at a) $x=-0.14 c$ and b) $x=-0.02 c$ and of the turbulence intensity TI at c) $x=-0.14 c$ and d) $x=-0.02 c:-$ present RANS, - RANS Christophe [34] and $\square$ experiment [61].

The comparison appears more favorable in the wake region, as can be seen in Fig. 13 showing the mean and rms streamwise and crosswise velocity components at $x=0.0574 c$. The present results are again in very good agreement with the RANS data reported by Christophe [34], and show much reduced discrepancies with the experimental data 
of ref. $[33,61]$. In particular, the present rms profiles predict the presence of two peaks located at $y=-0.015 c$ and $y=0.003 c$. Additionally, the width of the wake obtained in the present computation for $y>0$ is slightly larger than in the measurements, consistently with the boundary layer results reported in Fig. 12(a). Note that the good agreement that is found in the wake region concerns the velocity field, not the turbulence intensity as no turbulence measurement in the near-wake was available for comparison.
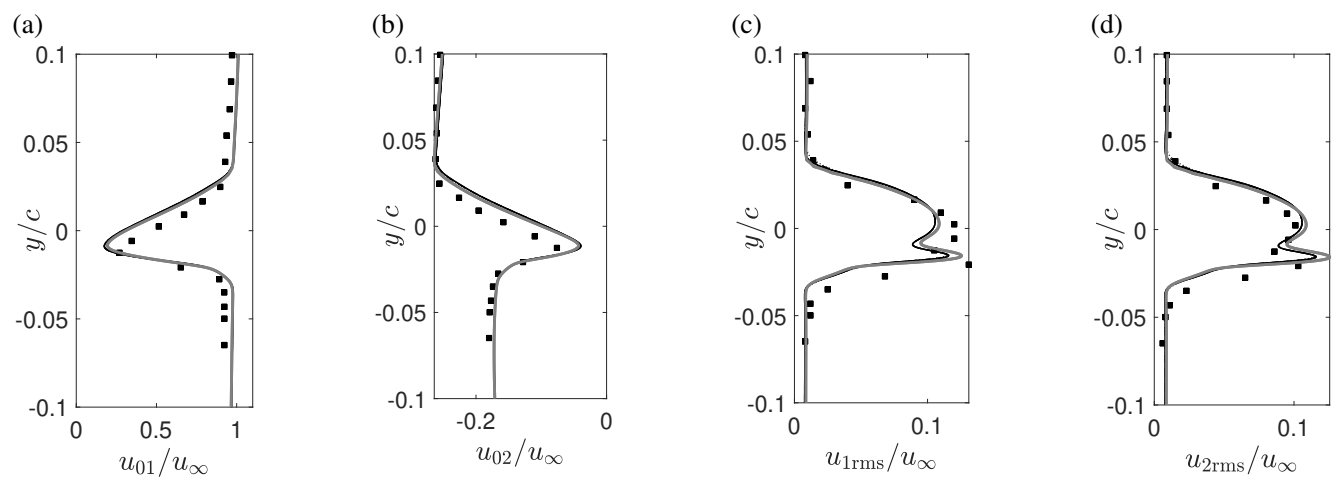

Fig. 13 Representation of the mean a) streamwise and b) crosswise velocities and the RMS c) streamwise and d) crosswise velocities at $x=0.0574 c$ : - present RANS, — RANS Christophe [34] and (ש) experiment [33, 61].

\section{B. Calculation of the sound sources using RPM and sound propagation using FEM}

In order to evaluate the applicability of the Helmholtz-based aeroacoustic approach for this flow configuration, both APE and Helmholtz FEM simulations are performed. Additionally, to examine the influence of the definition of the source term on acoustic spectra, a parametric study is conducted by varying the numerical parameters used in the RPM simulations of section IV.

\section{Set-up of RPM simulations}

The rectangular RPM domain $\Omega_{0}$ used in the simulations surrounds the trailing edge of the airfoil, as shown in Fig. 14. Its orientation is aligned with that of the chord. It has a size of $L_{x}=0.6 c$ and $L_{y}=0.2 c$, chosen to include the flow boundary layer and the high values of the turbulent kinetic energy found close to the trailing edge. According to the coordinate system of Fig. 14(b), the domain $\Omega_{0}$ extends from $-0.15 c$ to $0.45 c$ in direction $x_{c}$ and from $-0.08 c$ to $0.12 c$ in direction $y_{c}$, with the trailing edge located at $x_{c}=y_{c}=0$. The choice of the domain size is also motivated by an analysis of Christophe [34], based on an acoustic analogy framework, which states that most of the sound sources responsible for the trailing-edge noise of the $\mathrm{CD}$ airfoil are found in a region extending from a distance of $0.15 c$ upstream of the trailing-edge to a distance of $0.4 c$ downstream of the trailing-edge.

The frequency range of interest extends from $50 \mathrm{~Hz}$ to $3000 \mathrm{~Hz}$. The RPM simulations are carried out over a period $T=6310 c / c_{0}=2.52 \mathrm{~s}$ with a time step $\Delta t_{\mathrm{RPM}}$ chosen to satisfy the constraint Eq. (20). The velocity fluctuations are recorded at a sampling time $\Delta t_{\mathrm{ac}}=3.58 \times 10^{-1} \mathrm{c} / c_{0}=1.43 \times 10^{-4} \mathrm{~s}$, corresponding to the time step used to compute 
(a)

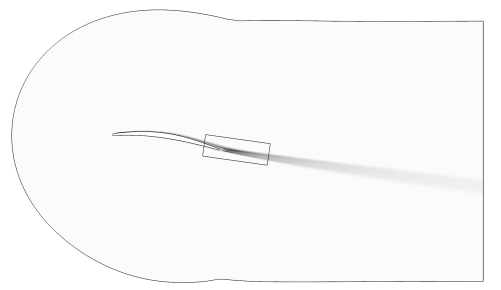

(b)

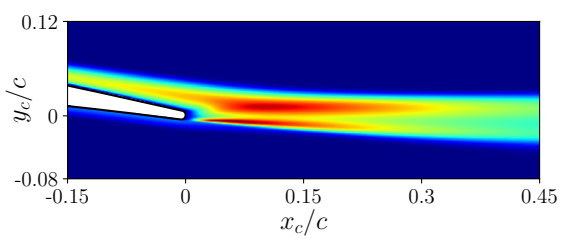

Fig. 14 a) RANS domain with rectangular RPM domain surrounding the trailing edge and b) RPM domain, colored by the turbulent kinetic energy obtained from RANS with levels extending from 0 to $0.02 u_{\infty}^{2}$.

the source term $\boldsymbol{L}^{\prime}$ up to $3500 \mathrm{~Hz}$ in the acoustic propagation (see section V.B.2). A number of time samples of about $N_{s}=1.76 \times 10^{4}$ is thus generated, which is found to be sufficient to ensure the convergence of the turbulence statistics in the vicinity of the airfoil trailing edge. All the RPM simulations have been done using a Gaussian filter threshold $\epsilon$ of $10^{-4}$, as suggested in the literature [13, 52]. The sensitivity of the aeroacoustic results with respect to the threshold $\epsilon$ has been examined by performing RPM simulations with values of $\epsilon$ of $10^{-4}, 10^{-3}$ and $10^{-2}$. For the present flow configuration, the turbulent kinetic energy profiles and the acoustic spectra are not affected by the variations of $\epsilon$. For the sake of conciseness, these results are not shown here. In the parametric study, the effect of the RPM grid resolution is examined in section V.B.3. The influence of the number of particles in domain $\Omega_{0}$ and the effect of the length scale parameter $l_{\text {factor }}$ are studied in sections V.B.6 and V.B.7.

The source term $\boldsymbol{L}^{\prime}$ is smoothly damped at the boundaries of the RPM domain $\Omega_{0}$ using the Hann window defined in Eq. (28). As for the NACA002 airfoil case presented in section IV, in direction $y$, the windowing ratio is of $y_{\mathrm{b}} / L_{y}=0.2$. At the left hand-side boundary in direction $x$, as the trailing edge is located in the left part of domain $\Omega_{0}$, a windowing extent of $0.08 L_{x}$ is used to avoid damping the sound sources near the trailing-edge. As the influence of the windowing extent is expected to be higher in the convection direction where the sources exit the domain, the effects of windowing in direction $x$ downstream of the trailing edge is investigated by performing RPM simulations for $x_{\mathrm{b}}=0.05 c, 0.15 c$ and $0.3 c$, corresponding to ratios $x_{\mathrm{b}} / L_{x}$ of $0.83,0.25$ and 0.5 (see section V.B.8).

\section{Set-up of FEM simulations}

For the FEM simulations, a 2-D circular computational domain of radius $1.5 c$ centered on the trailing edge of the airfoil is considered. The domain is represented in Fig. 15. At its external boundary, a four-layer PML region is used [39]. Similarly to the NACA0012 simulations, unstructured triangular meshes are designed using Gmsh [53], with quadratic elements of size $0.004 c$ at the airfoil walls. The largest elements, of size of $h_{\mathrm{ac}} \max =0.5 c$, are located at the PML interface. Near the trailing edge where the RPM sound sources are computed, the mesh is finer. A view of the mesh obtained for $h_{\text {ac source }}=0.01 c$, without the PML region, is presented in Fig. 15(b). The effect of the resolution of the FEM mesh in the source region is examined by performing acoustic simulations for $h_{\mathrm{ac}}$ source $=4 h_{\mathrm{RPM}}$, 
$h_{\mathrm{ac} \text { source }}=10 h_{\mathrm{RPM}}, 25 h_{\mathrm{RPM}}$ and $40 h_{\mathrm{RPM}}$ (see section V.B.4).

The Fourier components of the Lamb vector $\hat{\boldsymbol{L}}$, calculated from RPM, are interpolated on the FEM mesh using $p_{\text {source }}=10$, as described in section II.A.3. The RPM time signal $\boldsymbol{L}^{\prime}$ is divided into $N_{\text {seg }}=45$ segments of period $T_{\text {seg }}=208 c / c_{0}$ which overlap by $33 \%$, resulting in a frequency resolution of $\Delta f=1 / T_{\text {seg }}=4.8 \times 10^{-3} c_{0} / c=12 \mathrm{~Hz}$.

Thanks to the high-order adaptive FEM approach, the complete frequency range can be efficiently covered using a single input mesh. The a priori error indicator adjusts the polynomial order $p_{\text {FEM }}$ in each element and at each frequency so as to reach a user defined target accuracy $E_{T}$. As an illustration, the real part of the solution obtained for the first segment is shown for the highest frequency of interest, $f=3 \mathrm{kHz}$, in Figure 15(a). The associated face order distribution obtained in the FEM domain for the target accuracy $E_{T}=0.5 \%$ are represented in Fig 15(b). Near the airfoil and in the sound source region, the order of the solution is equal to $p_{\text {FEM }}=2$. As the distance from the trailing edge increases, the mesh gets coarser and the order increases accordingly. At $r=1.5 c$, an order $p_{\mathrm{FEM}}=5$ is used. In order to verify the convergence of the acoustic spectra, numerical tests have also been conducted for smaller values of $E_{T}$. For instance, the distribution of the face orders obtained at $f=3 \mathrm{kHz}$ for $E_{T}=0.01 \%$ is outlined in Fig. 15(c). As expected, higher orders are found in the acoustic region compared to the distribution obtained for $E_{T}=0.5 \%$, but no difference in the spectra was observed. Similarly, numerical tests have been performed by moving the PML interface further away from the source region, without any influence on the far-field spectra, hence qualifying the proposed PML configuration. These additional verification results are not reported here for conciseness.

(a)

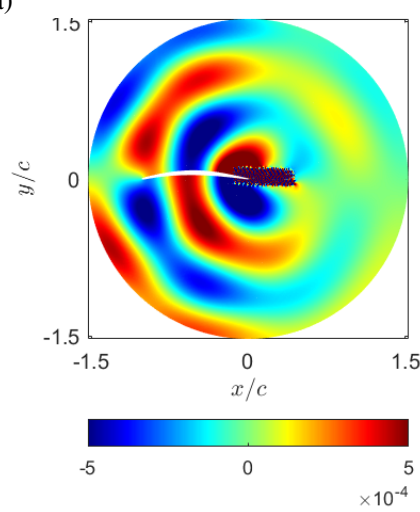

(b)

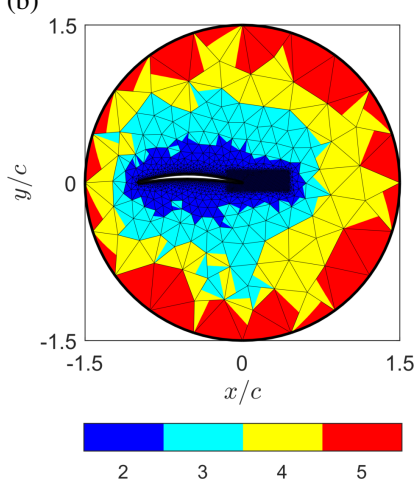

(c)

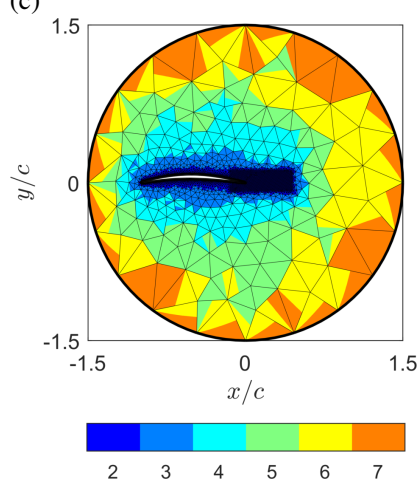

Fig. 15 Representation of (a) the real part of the acoustic pressure obtained at $f=3 \mathrm{kHz}$ and the acoustic mesh with $h_{\text {ac source }}=0.01 c$ colored by the FEM face orders obtained at $f=3 \mathrm{kHz}$ for $(\mathrm{b}) E_{T}=0.5 \%$ and (c) $E_{T}=0.01 \%$.

As in the experiment [32], the sound levels in the far-field region are computed at a distance of $r_{\mathrm{obs}}=2 \mathrm{~m}$ from the trailing-edge. For this purpose, as in section IV, the acoustic data are recorded at the PML interface and a 2-D integral method is used to determine the values of $\hat{p}$ in the far-field region. The values of $\hat{p}$ obtained for the 45 segments are then averaged to compute the power spectral density $S_{p p}$. 
The 3-D sound predictions are computed from the 2-D simulations using the 2-D to 3-D correction given in Eq. (29). The SPL are are integrated in bins of width $\Delta f_{\exp }=8 \mathrm{~Hz}$ to make them comparable with the experimental results [32]. As for the NACA0012 case, a calibration offset of $-6.5 \mathrm{~dB}$ has been applied to all the acoustic spectra presented in this section.

\section{Effect of the RPM grid resolution}

Helmholtz-based simulations are performed to examine the influence of the RPM grid using four meshes referred to as VeryCoarseGrid, CoarseGrid, MediumGrid and FineGrid. The meshes are characterized by a constant grid spacing $h_{\mathrm{RPM}}$ of $0.004 c, 0.002 c, 0.001 c$ and $0.0005 c$, respectively. The parameters of the simulations are reported in Tab. 1 . While the time step $\Delta t_{\mathrm{RPM}}$ is chosen so that $\Delta t_{\mathrm{RPM}}=0.6 h_{\mathrm{RPM}} / \max \left(\left|\boldsymbol{u}_{0}(\boldsymbol{x})\right|\right)$, the sampling time $\Delta t_{\mathrm{ac}}$ is kept constant and is of about $0.35 c / c_{0}$. The simulations are performed using 2.5 particles per RPM grid cell, a length scale factor $l_{\text {factor }}$ of 2 , a windowing function extending over $0.15 c$ in the downstream direction and an acoustic grid spacing $h_{\mathrm{ac}}$ source of $0.01 c$ in the source region.

\begin{tabular}{llllllll}
\hline \hline RPM grid & $N_{\mathrm{ppc}}$ & $l_{\text {factor }}$ & $h_{\mathrm{RPM}}$ & $\Delta t_{\mathrm{RPM}}$ & $\Delta t_{\mathrm{ac}}$ & $x_{b \text { downstream }}$ & $h_{\mathrm{ac}}$ source \\
\hline \hline VeryCoarseGrid & 2.5 & 6 & $0.004 c$ & $4.78 \times 10^{-2} c / c_{0}$ & $7 \Delta t_{\mathrm{RPM}}$ & $0.15 c$ & $0.01 c$ \\
\hline CoarseGrid & 2.5 & 6 & $0.002 c$ & $2.39 \times 10^{-2} c / c_{0}$ & $15 \Delta t_{\mathrm{RPM}}$ & $0.15 c$ & $0.01 c$ \\
\hline MediumGrid & 2.5 & 6 & $0.001 c$ & $1.19 \times 10^{-2} c / c_{0}$ & $30 \Delta t_{\mathrm{RPM}}$ & $0.15 c$ & $0.01 c$ \\
\hline FineGrid & 2.5 & 6 & $0.0005 c$ & $5.97 \times 10^{-3} c / c_{0}$ & $60 \Delta t_{\mathrm{RPM}}$ & $0.15 c$ & $0.01 c$ \\
\hline \hline
\end{tabular}

Table 1 Parameters of the simulations in the study of the sensitivity of the RPM grid.

Snapshots of the amplitude of the Lamb vector components obtained using VeryCoarseGrid, MediumGrid and FineGrid are represented in Fig. 16. For the three meshes, the amplitude of the streamwise component of the Lamb vector is higher than the spanwise component by one order of magnitude, as expected. Using VeryCoarseGrid, only large scale components are discretized. Using finer grids, the Lamb vector components also display smaller scales.

The turbulent kinetic energy synthetized in the RPM domain is compared with the RANS turbulent kinetic energy in Fig. 17. In the RANS results, two distinct lobes are observed in the wake of the airfoil. In the RPM simulation using VeryCoarsegrid, poor kinetic energy levels are predicted. Using Coarsegrid, only the upper lobe is retrieved. For finer meshes, two lobes are captured but the energy levels remain lower than the ones predicted by RANS.

More quantitative results about the turbulence statistics are presented in Figs. 18(a) and 18(b) where the turbulent kinetic energy profiles obtained from the four RPM simulations at $x_{c}=-0.02 c$ and $x_{c}=0.1 c$ are compared with the RANS profiles. Using VeryCoarseGrid, the energy levels are poorly predicted. Using CoarseGrid, higher levels are obtained except near the walls and in the wake. In these regions, the resolution of CoarseGrid is not fine enough to accurately predict the turbulence levels. As the mesh is refined, a better agreement is found with the RANS data. However, as already observed for the NACA cases in section IV, the energy peaks predicted by RPM are smoother than 
(a)

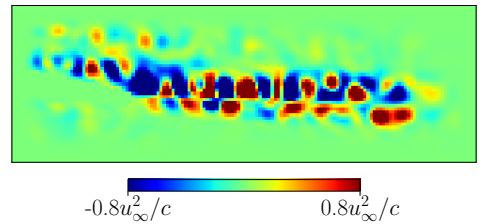

(d)

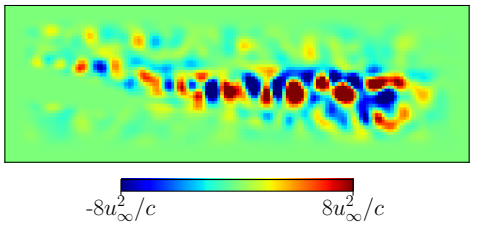

(b)

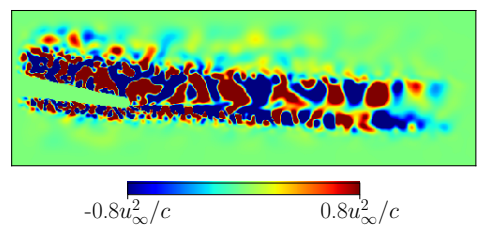

(e)

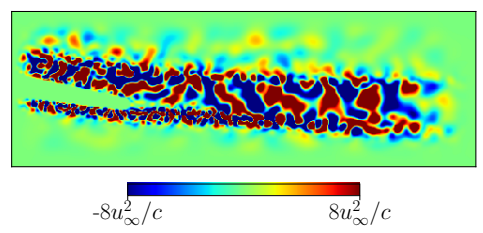

(c)

(f)
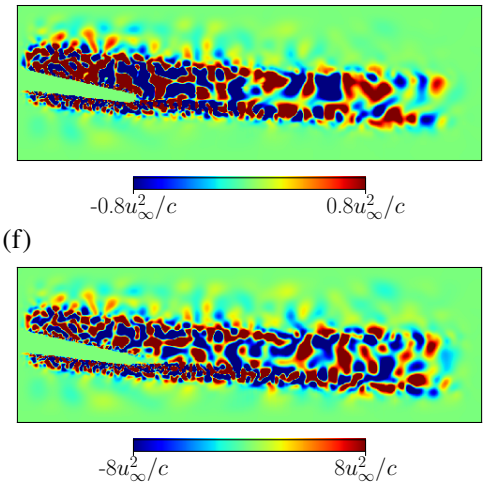

Fig. 16 Snapshots of the Lamb vector components in domain $\Omega_{0}: L_{x}^{\prime}$ for (a) VeryCoarseGrid, (b) MediumGrid, (c) FineGrid and $L_{y}^{\prime}$ for (d) VeryCoarseGrid, (e) MediumGrid, (f) FineGrid.

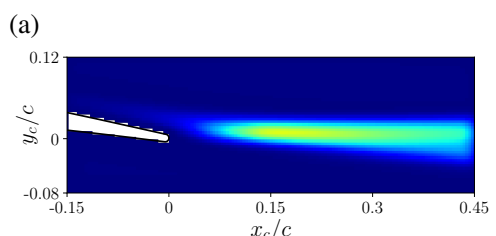

(d) (b)

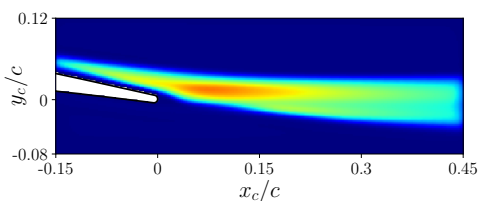

(e) (c)

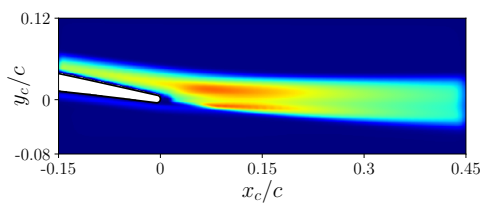

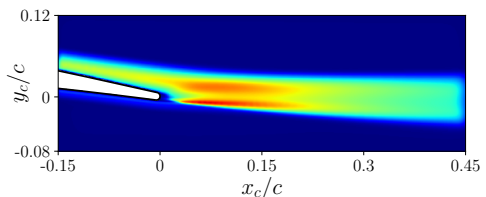

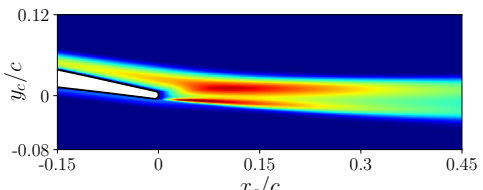

Fig. 17 Representation of the turbulent kinetic energy in domain $\Omega_{0}$ : RPM results using (a) VeryCoarseGrid, (b) CoarseGrid, (c) MediumGrid, (d) FineGrid and (e) RANS results. The color scale is from 0 to $0.02 u_{\infty}^{2}$.

the RANS ones.

The sound pressure levels obtained at $r_{\mathrm{obs}}=2 \mathrm{~m}$ and $\theta_{\mathrm{obs}}=90^{\circ}$ with the source terms calculated from the four RPM grids are presented in Fig. 18(c). Broadband spectra characterized by a sharp decrease of the sound levels at a frequency of about $f=1 \mathrm{kHz}$ are obtained. Using FineGrid and MediumGrid, similar sound spectra are obtained. Their shape is also in agreement with the experimental one up to $1 \mathrm{kHz}$. For $f>1 \mathrm{kHz}$, the fast decrease of the amplitude of the RPM spectra is most likely due to the Gaussian energy spectrum used in this work to model the turbulence in the RPM simulation [46]. A more realistic model such as a von Kármán energy spectrum could be used in order to improve the acoustic predictions at these frequencies, but at the price of a higher computational cost. Using CoarseGrid, the sound levels at $f>1 \mathrm{kHz}$ are lower than those calculated with finer RPM grids. Using VeryCoarseGrid, the acoustic levels are underestimated over the entire frequency range. This can be related to the poor reconstruction of the turbulent kinetic energy field that is observed in Fig. 17 for the two coarsest grids. 
(a)

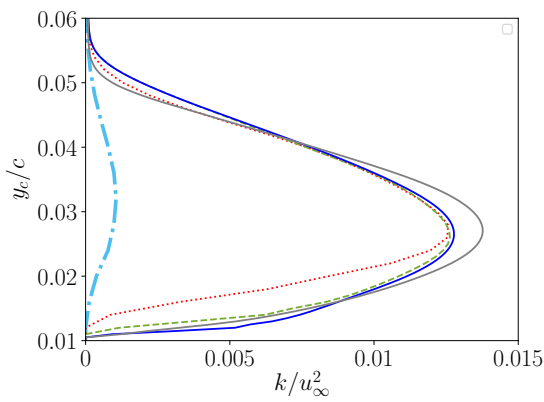

(b)

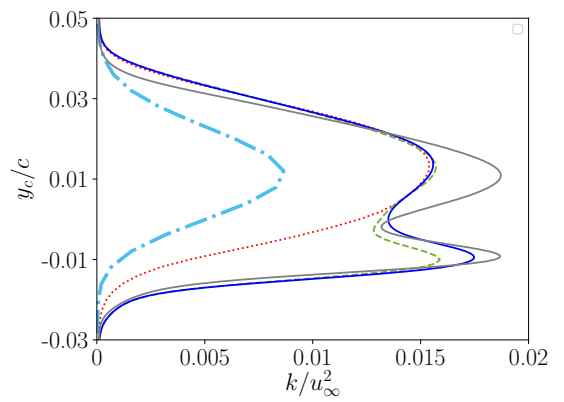

(c)

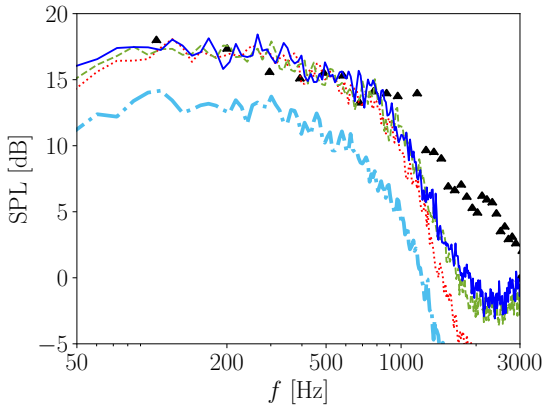

Fig. 18 Representation of the turbulent kinetic energy at (a) $x_{c}=-0.02 c$ and (b) $x_{c}=0.1 c$, and (c) SPL at $r_{\text {obs }}=2 \mathrm{~m}$ and $\theta_{\text {obs }}=90^{\circ}$ : RPM-FEM using $\cdots$ VeryCoarseGrid, $\cdots$ CoarseGrid, -- MediumGrid, — FineGrid, — RANS and $\triangle$ experiment [32].

\section{Effect of the acoustic grid resolution in the sound source region}

The effect of the resolution of the acoustic grid in the source region is examined by performing FEM simulations with the Helmholtz solver for four acoustic meshes.A first acoustic mesh is designed with the ratio $h_{\text {ac source }} / h_{\mathrm{RPM}}=4$ used for the NACA0012 problem yielding $h_{\mathrm{ac}}$ source $=0.004 c$. Three coarser meshes are then defined. They are characterized by $h_{\text {ac source }}=0.01 c, 0.025 c$ and $0.04 c$ corresponding to $10 h_{\mathrm{RPM}}, 25 h_{\mathrm{RPM}}$ and $40 h_{\mathrm{RPM}}$. The four acoustic grids in the vicinity of the airfoil are represented in Fig 19. While the mesh resolution is varied in the sound source region, it is kept constant at the airfoil wall with quadratic elements of size $0.004 c$ in order to properly represent the airfoil geometry. The four meshes also have the same resolution near the PML interface, with $h_{\mathrm{ac}} \max =0.5 c$. The RPM simulation is performed using the mesh MediumGrid and the parameters $N_{\mathrm{ppc}}=2.5, l_{\text {factor }}=6$ and $x_{b}$ downstream $=0.15 c$. At $f=3 \mathrm{kHz}$, the number of degrees of freedom in the FEM simulations is of $1.19 \times 10^{4}$ for $h_{\text {ac source }}=0.04 c$, of $1.23 \times 10^{4}$ for $h_{\text {ac source }}=0.025 c$, of $1.70 \times 10^{4}$ for $h_{\text {ac source }}=0.01 c$ and of $4.25 \times 10^{4}$ for $h_{\text {ac source }}=0.004 c$.

(a)

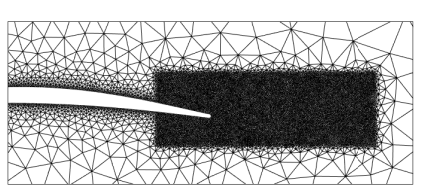

(b)

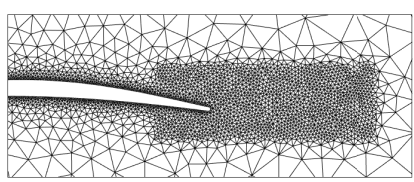

(d)

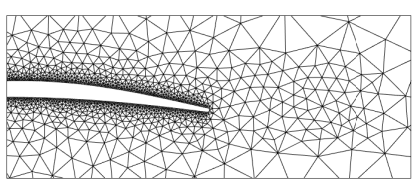

(c)

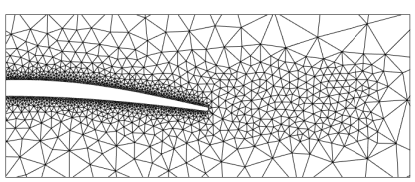

Fig. 19 Representation of the acoustic grids: (a) $h_{\text {ac source }}=0.004 c,(b) h_{\text {ac source }}=0.01 c,(c) h_{\text {ac source }}=0.025 c$ and (d) $h_{\text {ac source }}=0.04 c$.

The SPL obtained with the four grids at $r_{\mathrm{obs}}=2 \mathrm{~m}$ and $\theta_{\mathrm{obs}}=90^{\circ}$ are presented in Fig. 20, as a function of the frequency. Using the coarsest acoustic grid with $h_{\mathrm{ac}}$ source $=0.04 c$, higher sound levels are obtained, especially for $f>1 \mathrm{kHz}$ where spurious noise is produced. A good agreement is reported between the acoustic spectra obtained 
using the three finest meshes indicating that the acoustic mesh is fine enough for $h_{\mathrm{ac}}$ source $<0.025 c$ for the present flow configuration. In the following, the FEM simulations are all performed using $h_{\mathrm{ac}}$ source $=0.01 c$.

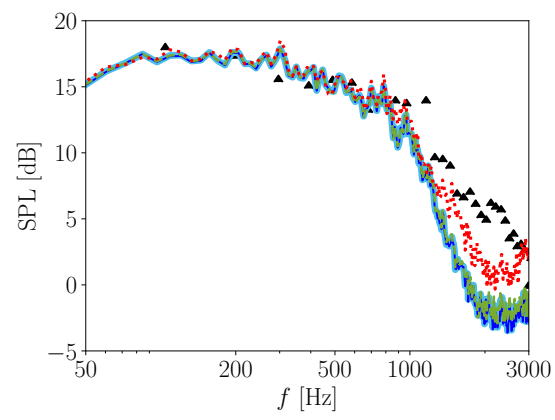

Fig. 20 SPL at $r_{\mathrm{obs}}=2 \mathrm{~m}$ and $\theta_{\mathrm{obs}}=90^{\circ}:$ RPM-FEM using $\boldsymbol{h}_{\text {ac source }}=0.004 c-h_{\text {ac source }}=0.01 c$, $--h_{\text {ac source }}=0.025 c \cdots h_{\text {ac source }}=0.04 c$ and $\Delta$ experiment [32].

\section{Effect of the mean flow}

The sensitivity of the RPM and FEM grids being examined, the effect of the mean flow in the acoustic propagation problem is studied by performing a FEM simulation solving the APE in the frequency domain using the acoustic grid with $h_{\text {ac source }}=0.01 c$. The mean flow data obtained from RANS are interpolated onto the FEM mesh using the same technique as presented in section IV. For RPM, the mesh MediumGrid and the parameters $N_{\text {ppc }}=2.5, l_{\text {factor }}=6$ and $x_{b \text { downstream }}=0.15 c$ are used. The SPL obtained with the Helmholtz and the APE approaches at $r_{\mathrm{obs}}=2 \mathrm{~m}$ and $\theta_{\mathrm{obs}}=90^{\circ}$ are represented in Fig. 21(a). The sound directivities at $r_{\mathrm{obs}}=2 \mathrm{~m}$ and $f=400 \mathrm{~Hz}$ are plotted in $\mathrm{dB}$ in Fig. 21(b). The FEM and APE results overlap which confirms that the convection and refraction effects of the mean flow can be neglected for this flow configuration.

(a)

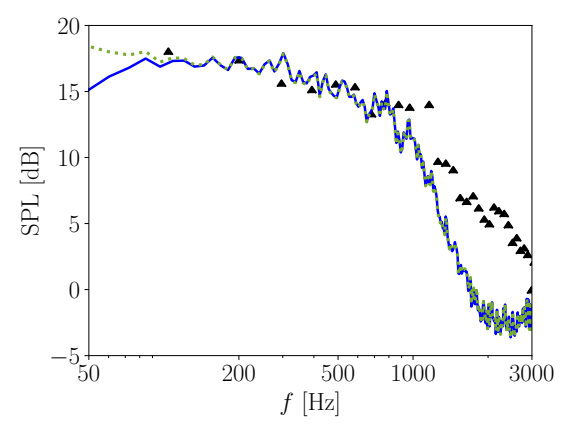

(b)

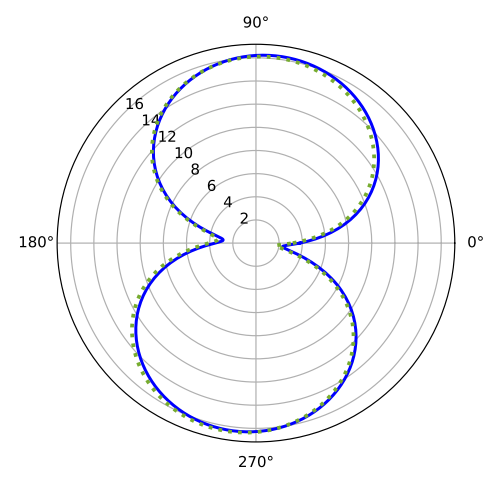

Fig. 21 Representation of (a) the SPL at $r_{\mathrm{obs}}=2 \mathrm{~m}$ and $\theta_{\mathrm{obs}}=90^{\circ}$ and (b) the sound directivity at $r_{\mathrm{obs}}=2 \mathrm{~m}$ at $f=400 \mathrm{~Hz}$ :— Helmholtz solver and - - APE-4 solver. 


\section{Effect of the number of particles in the RPM domain}

The influence of the number of particles used for RPM is studied by performing simulations with MediumGrid for $N_{\text {ppc }}=0.25,2.5$ and 10. The other simulation parameters are listed in Tab. 2, including $l_{\text {factor }}=6, x_{b}$ downstream $=0.15 c$ and $h_{\mathrm{ac}}=0.01 c$. Surprisingly, the turbulent kinetic energy profiles obtained at $x_{c}=-0.02 c$ and $x_{c}=0.1 c$ are not significantly affected by variations of the number of particles in the RPM domain, and the results are not displayed here. The sound spectra calculated at $r_{\mathrm{obs}}=2 \mathrm{~m}$ and $\theta_{\mathrm{obs}}=90^{\circ}$ are represented in Fig. 22. Overall, the effect of $N_{\mathrm{ppc}}$ on the sound levels is marginal and no clear convergence with respect to the number of particles is reported.

\begin{tabular}{lllll}
\hline \hline RPM grid & $N_{\mathrm{ppc}}$ & $l_{\text {factor }}$ & $x_{b \text { downstream }}$ & $h_{\text {ac source }}$ \\
\hline \hline MediumGrid & $\mathbf{0 . 2 5}$ & 6 & $0.15 c$ & $0.01 c$ \\
\hline MediumGrid & $\mathbf{2 . 5}$ & 6 & $0.15 c$ & $0.01 c$ \\
\hline MediumGrid & $\mathbf{1 0}$ & 6 & $0.15 c$ & $0.01 c$ \\
\hline \hline
\end{tabular}

Table 2 Parameters of the simulations in the study of the influence of the number of particles in RPM domain.

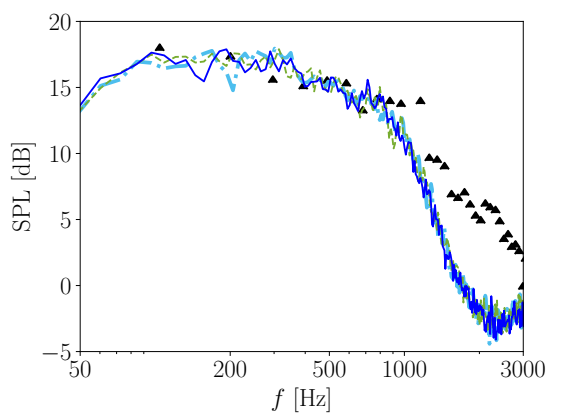

Fig. 22 Representation of the SPL at $r_{\mathrm{obs}}=2 \mathrm{~m}$ and $\theta_{\mathrm{obs}}=90^{\circ}$ : (a) RPM-FEM using $N_{\mathrm{ppc}}=0.25$, $--N_{\text {ppc }}=2.5,-N_{\text {ppc }}=10$ and $\triangle$ experiment [32].

\section{Effect of the length scale parameter $l_{\text {factor }}$}

To study the effect of the parameter $l_{\text {factor }}$ appearing in Eq. (19) and defining the width of the RPM filter (16), three Helmholtz-based simulations are carried out for $l_{\text {factor }}=4,6$ and 8 . The simulation settings are given in Tab. 3. The results on the reconstructed turbulent kinetic energy at $x_{c}=-0.02 c$ and $x_{c}=0.1 c$ are shown in Figs. 23(a) and 23(b). At $x_{c}=-0.02 c$, the turbulence levels predicted by RPM get closer to the target RANS value as the value of $l_{\text {factor }}$ decreases. A possible explanation is that when the length scale gets smaller, the support of a particle (i.e. a square of half-width 2.43 $\Lambda$ ) is reduced. Consequently, the variation of the RANS turbulence statistics over this support is expected to be reduced as well. As in the RPM method, the less the turbulence properties vary spatially the better the RPM reconstruction, a better agreement between the target and the effective statistics is obtained. At $x_{c}=0.1 c$, an improved reconstruction of the kinetic energy levels is also observed for $y_{c}>0$ when $l_{\text {factor }}$ decreases. On the contrary, for $y_{c}<0$, the length scale value obtained using $l_{\text {factor }}=4$ shows the largest discrepancies with the RANS profile. 
This is attributed to the length scale value obtained using $l_{\text {factor }}=4$ which is smaller than $\Lambda_{\min }=4 h_{\mathrm{RPM}}$ and thus not accurately represented by the RPM grid. The effect of the length scale parameter $l_{\text {factor }}$ on the far-field SPL is presented in Fig. 23(c). The sound levels increase with the value of $l_{\text {factor }}$, as also observed in Ref. [26]. This can be explained by the larger radiation efficiency of larger scales. But in contrast with the effect of the RPM grid resolution discussed in Section V.B.3, a change in the value of $l_{\text {factor }}$ systematically affects all frequencies including the lowest ones. These results highlight the difficulty to properly predict the amplitude of the sound spectra using this sound synthesis technique, especially as there is no consensus on the choice of the empirical parameter $l_{\text {factor }}$ in literature.

\begin{tabular}{lllll}
\hline \hline RPM grid & $N_{\mathrm{ppc}}$ & $l_{\text {factor }}$ & $x_{b \text { downstream }}$ & $h_{\mathrm{ac} \text { source }}$ \\
\hline \hline MediumGrid & 2.5 & $\mathbf{4}$ & $0.15 c$ & $0.01 c$ \\
\hline MediumGrid & 2.5 & $\mathbf{6}$ & $0.15 c$ & $0.01 c$ \\
\hline MediumGrid & 2.5 & $\mathbf{8}$ & $0.15 c$ & $0.01 c$ \\
\hline \hline
\end{tabular}

Table 3 Parameters of the simulations in the study of the influence of the length scale parameter $l_{\text {factor }}$.

(a)

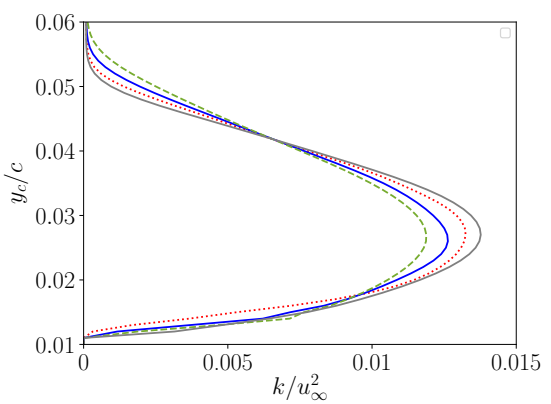

(b)

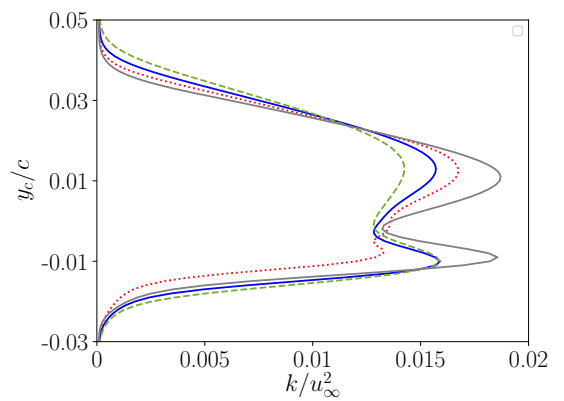

(c)

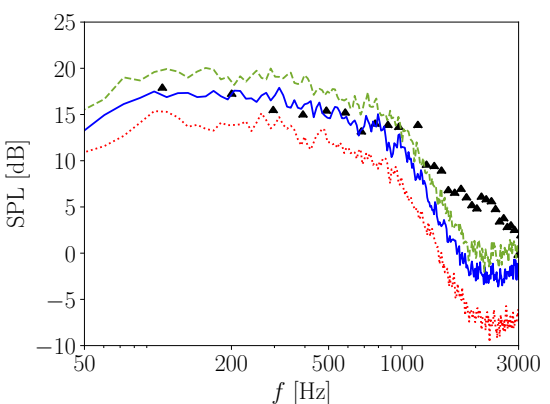

Fig. 23 Representation of the turbulent kinetic energy at (a) $x_{c}=-0.02 c$ and (b) $x_{c}=0.1 c$, and (c) SPL at $r_{\text {obs }}=2 \mathrm{~m}$ and $\theta_{\text {obs }}=90^{\circ}:$ RPM-FEM using $\cdots l_{\text {factor }}=4,-l_{\text {factor }}=6,---l_{\text {factor }}=8$, - RANS and $\Delta$ experiment [32].

\section{Effect of the window extent downstream of the trailing edge}

The influence on the acoustic results of the extent of the window at the right boundary of the RPM domain is examined by performing simulations with $x_{\mathrm{b} \text { downstream }}=0.05 c, 0.15 c$ and $0.3 c$. The RPM and FEM simulations are carried out using the RPM mesh MediumGrid, the FEM Helmholtz solver and the parameters $N_{\mathrm{ppc}}=2.5, l_{\text {factor }}=6$ and $h_{\text {acsource }}=0.01 c$. The windowing function obtained for each value of $x_{\mathrm{b}}$ downstream is plotted as a function of the streamwise direction in Fig. 24(a). The sound levels at $r_{\mathrm{obs}}=2 \mathrm{~m}$ and $\theta_{\mathrm{obs}}=90^{\circ}$ are presented in Fig. 24(b). The length over which the window is applied strongly influences the shape of the acoustic spectrum. Using $x_{\mathrm{b}}$ downstream $=0.05 c$, the spatial windowing is too sharp to efficiently remove spurious radiation from the downstream source region for

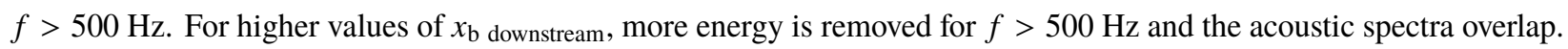

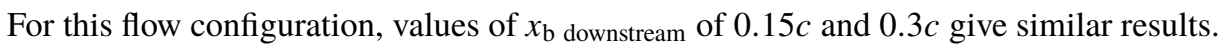


(a)

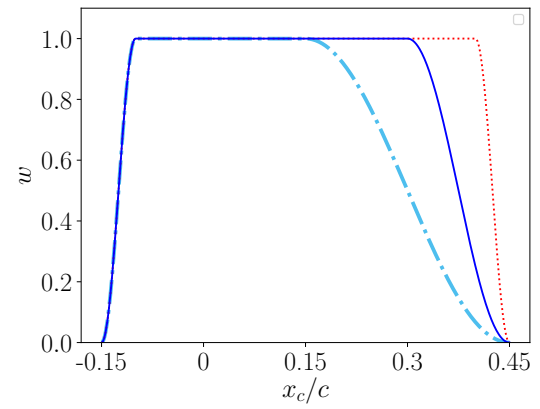

(b)

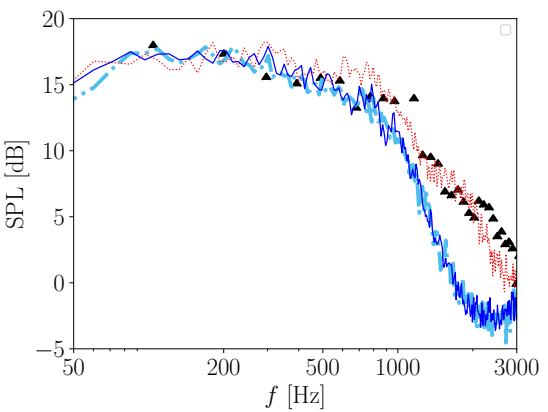

Fig. 24 Representation of (a) the windowing function in the streamwise direction and (b) the SPL at $r_{\mathrm{obs}}=2 \mathrm{~m}$ and $\theta_{\mathrm{obs}}=90^{\circ}$ : RPM-FEM using $\cdots x_{\mathrm{b} \text { downstream }}=0.05 c,-x_{\mathrm{b} \text { downstream }}=0.15 c,-x_{\mathrm{b} \text { downstream }}=0.3 \mathrm{c}$ and $\triangle$ experiment [32].

\section{Computational cost and data storage}

In the RPM simulations, the computational cost at each time iteration is mainly driven by the resolution of the RPM grid and the number of particles. In the FEM simulations, performed using an in-house adaptive $p$-FEM code written in Matlab, the computational cost is mainly driven by the assembly of the right-hand sides. It drastically increases for large number of right-hand sides and fine resolutions of the acoustic grid in the sound source region. All the run times reported hereafter have obtained using a laptop Intel Core i7 running at $2.6 \mathrm{GHz}$ with $64 \mathrm{~GB}$ of memory.

\section{RPM simulations}

The run times of the RPM simulations performed for the CD airfoil case are listed in Tab. 1 are reported in Fig. 25(a) in logarithmic scale as a function of the normalized RPM grid spacing $h_{\mathrm{RPM}} / c$. The RPM simulations last from $0.05 \mathrm{~h}$ up to $16.7 \mathrm{~h}$. The RPM computational time increases with the number of RPM grid cells. A best-fit slope $\left(h_{\mathrm{RPM}} / c\right)^{-3}$ is reported. The RPM run time is represented as a function of the number of particle(s) per grid cell $N_{\mathrm{ppc}}$ in Fig. 25(b). The simulations have been done using RPM mesh MediumGrid, and the length scale parameter $l_{\text {factor }}=6$. The computational cost increases with $N_{\mathrm{ppc}}$. It varies from $0.9 \mathrm{~h}$ using $N_{\mathrm{ppc}}=0.05$ up to $5.6 \mathrm{~h}$ using $N_{\mathrm{ppc}}=10$. The cost associated to the RPM simulation could be further reduced by the implementation of a multi-threaded version of the $\mathrm{C}++$ code. The storage of the RPM sound source term used in the FEM simulations leads to an amount of data varying from 2.4 GB for VeryCoarseGrid up to 137 GB for FineGrid.

\section{FEM simulations}

The performance of the APE and Helmholtz solvers are provided in Tab. 4 in terms of number of degrees of freedom (DoFs), memory footprint and run times for the two airfoil configurations at $f=3 \mathrm{kHz}$. The solve time reported by the embedded sparse linear solver MUMPS [29] includes the factorization and forward-backward substitutions for the right-hand-sides (one per time segment). For the $\mathrm{CD}$ airfoil case, the results obtained using the acoustic grid with 
(a)

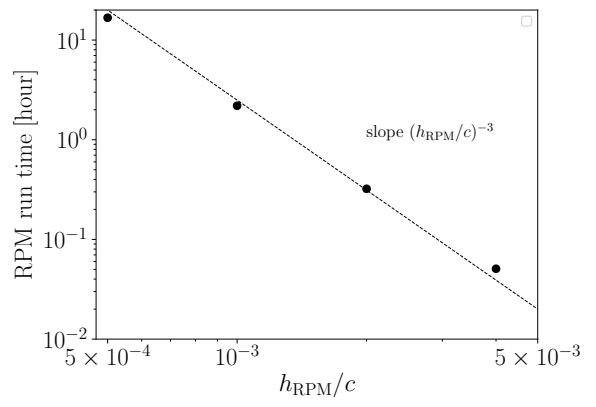

(b)

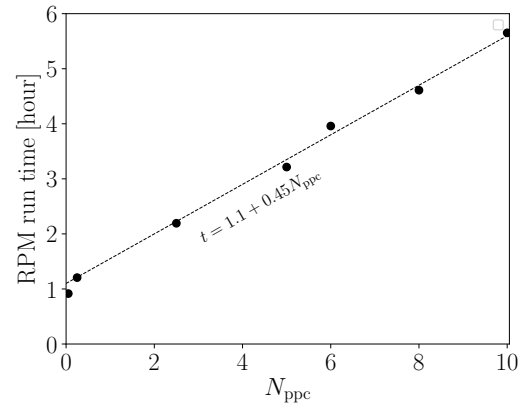

Fig. 25 Representation of the computational cost of the RPM simulation for the CD airfoil case as a function of (a) the RPM grid spacing and (b) the number of particles per RPM grid cell $N_{\text {ppc }}$.

$h_{\text {ac source }}=0.01 c$ are reported.

The use of the Helmholtz solver reduces the memory footprint of the simulations by more than an order of magnitude compared to an APE solver.

The reported run times for the system assembly are costly and dominated by the assembly of the right-hand sides, which involves multiple source evaluations in the refined source region. Using the Helmholtz solver, it is of approximately $30 \mathrm{~s}$ for the CD airfoil and of $140 \mathrm{~s}$ for the NACA0012 airfoil. The assembly time is multiplied by a factor of roughly 2 using the APE solver. It is worth emphasizing that the simulations are performed using Matlab on a single thread, and that therefore, considerably lower assembly timings could be obtained with an efficient $\mathrm{C}++$ parallel implementation of the proposed approach.

By contrast, the solve time reported by MUMPS solver is relatively low. Using the Helmholtz solver, it is of $0.5 \mathrm{~s}$ for the CD airfoil case and of $2.3 \mathrm{~s}$ for the NACA0012 airfoil case. An overhead factor of approximately 2 on the execution time is reported using the APE solver.

\begin{tabular}{llllll}
\hline \hline configuration & method & $\begin{array}{l}\text { number of } \\
\text { Dofs }\end{array}$ & $\begin{array}{l}\text { Matlab } \\
\text { assembly time }\end{array}$ & $\begin{array}{l}\text { MUMPS } \\
\text { solve time }\end{array}$ & $\begin{array}{l}\text { MUMPS } \\
\text { factorization memory }\end{array}$ \\
\hline \hline CD airfoil $\left(h_{\mathrm{ac}}=0.01 c\right)$ & Helmholtz & 17000 & $30 \mathrm{~s}$ & $0.5 \mathrm{~s}$ & $31 \mathrm{MB}$ \\
\hline CD airfoil $\left(h_{\mathrm{ac}}=0.01 c\right)$ & APE & 51600 & $60 \mathrm{~s}$ & $1 \mathrm{~s}$ & $273 \mathrm{MB}$ \\
\hline NACA0012 & Helmholtz & 85800 & $140 \mathrm{~s}$ & $2.3 \mathrm{~s}$ & $150 \mathrm{MB}$ \\
\hline NACA0012 & APE & 264500 & $290 \mathrm{~s}$ & $5.7 \mathrm{~s}$ & $1681 \mathrm{MB}$ \\
\hline
\end{tabular}

Table 4 Computational costs and memory footprint obtained from the MUMPS linear solver [29] at $f=3 \mathrm{kHz}$.

\section{Conclusions}

In this study, a computational aeroacoustic approach based on the combination of a Helmholtz solver and a stochastic approach has been proposed for low Mach number flow applications, for which the acoustic propagation is not significantly affected by convection nor refraction effects. Using a Helmholtz solver in place of a more computationally 
expensive APE solver, the memory footprint of the acoustic simulation is reduced by an order of magnitude and the run time is divided by a factor of about 2 without significantly impacting the sound predictions.

The source synthesis and acoustic propagation have been performed in two dimensions and a three-dimensional acoustic field has been obtained through an a posteriori correction. This also minimizes the computational effort while remaining relevant to flows with a certain spanwise homogeneity. The first purpose of this paper was to verify the prediction accuracy of this simulation strategy by application to a synthetic scalar source case. The second objective was to evaluate the applicability of the methodology to airfoil trailing-edge noise problems, in particular with regard to neglecting the mean flow effects for sound propagation. The third objective was to provide a sensitivity analysis of the numerical solution with regard to key user-defined parameters of the simulation procedure.

The sound sources have been generated on a uniform Cartesian grid using a time-domain RPM solver, and spatially-filtered to match the correlation length inferred from the local RANS turbulent kinetic energy and dissipation rate. In this work, a Gaussian distribution of energy over the turbulent scales was adopted. For trailing-edge noise problems, the equivalent sources, in this case represented by the Lamb vector, were Fourier-transformed and interpolated on the unstructured mesh used in a high-order finite element acoustic solver. A gradual spatial windowing was applied in order to provide a smooth decay of the acoustic sources at the boundaries of the Cartesian grid and thus minimize spurious noise.

The performance of the RPM-Helmholtz approach has been first verified for a Gaussian-distributed scalar source case. The numerical predictions were shown to be in good agreement with the analytical results, indicating that the RPM method reproduced the target turbulence properties accurately and that the Helmholtz solver matched the analytical solution.

The RPM-Helmholtz approach was then applied to predict the sound field radiated from the trailing edge of a NACA0012 airfoil for two flow configurations characterized by Mach numbers of 0.1118 and 0.1664 . The sound source synthesis was performed following best practices found in the literature. Numerical simulations performed with the Helmholtz and APE solvers have been compared against experimental data from the literature. The mean flow effect has no strong influence on the acoustic predictions which suggests that the Helmholtz-based methodology can be applied to low Mach number flow configurations. The shape of the acoustic spectra obtained numerically are in fair agreement with reference data up to $5 \mathrm{kHz}$, but a calibration offset is needed to match the absolute levels measured experimentally, as often reported in the literature. The methodology is then applied to a controlled-diffusion airfoil for a flow at a Mach number of 0.047. The results of the numerical simulations carried out with the Helmholtz and APE solvers indicate that the mean flow has no influence on the sound propagation. The shape of the far-field sound spectra is close to that of the experimental spectrum from the literature, up to a frequency of about $1 \mathrm{kHz}$. A more rapid tail-off of the spectrum was obtained at higher frequencies, which can be explained by the adoption of a spatial filter corresponding to a Gaussian distribution of energy across the wavelengths. The implementation of a more realistic spectrum, such as those of von 
Kármán or Liepmann, is likely to yield a more accurate decay at high frequencies and is left for future developments.

A parametric study has also been conducted on the controlled-diffusion airfoil case in order to evaluate the effect of simulations parameters including the size of the Cartesian RPM grid cells and of the acoustic grid cells, the number of particles per cell, the width of the filtering kernel, and the extent of the weighting window used to smooth the sound sources downstream of the airfoil trailing-edge. The most striking result is the influence of the width of the Gaussian spatial filter, defined as a function of the length scale empirical parameter $l_{\text {factor }}$, which was shown to affect the sound levels over the complete frequency range. This could partly explain the difficulty in predicting the absolute sound levels using this stochastic approach. The results were shown to be remain robust for relatively low values of the number of particles per RPM cell compared with the literature. Concerning the RPM Cartesian grid cells, the recommended grid spacing of $1 / 2000^{\text {th }}$ of the chord yields satisfactory results, but could be larger and up to $1 / 1000^{\text {th }}$ for this controlled-diffusion airfoil case. Concerning the effect of the acoustic mesh resolution in the sound source region, it was shown that the application of a high-order mapping of the sources allowed the use of acoustic cells up to 25 times larger than the RPM ones, without any significant loss of accuracy. A ratio of only 4 was identified here for the NACA0012 case. The numerical errors manifest themselves as a spurious increase of the acoustic levels, predominantly at high frequencies. A similar type of contamination was observed when the size of the weighting window was not sufficiently large.

As a final remark, it should be kept in mind that the recommended values reported for each parameter in this paper are likely to depend strongly upon the flow configuration, in this instance a flow where the noise generation is concentrated in the immediate neighborhood of the geometrical singularity represented by the trailing edge. In terms of future work, the use of more realistic turbulence spectra is certainly interesting, but the priority should be first to obtain robust predictions of the absolute sound levels by providing general guidelines to choose the RPM method parameters such as $l_{\text {fac }}$. Another direction of research would be to compute sources of trailing-edge noise from high-fidelity CFD results (such as Large-Eddy Simulations) instead of RPM. While very costly, this would provide useful information regarding the ability of synthetic turbulence to represent the true noise sources in this application.

\section{Appendix}

\section{A. Gaussian-distributed scalar source. Effect of the number of time segments.}

The convergence of the SPL profiles with respect to the simulation time is examined by performing four RPM-FEM simulations of periods $T$ of $200 \ell / c_{0}, 400 \ell / c_{0}, 800 \ell / c_{0}$ and $3200 \ell / c_{0}$, respectively. Each time signal is then divided into time segments of duration $20 \ell / c_{0}$ which overlap by $50 \%$. It yields a number of time segments $N_{\text {seg }}$ of $19,39,79$ and 319. The SPL obtained from the three RPM-FEM simulations at $r=1.5 \ell$ and $\theta=0^{\circ}$ are represented in Fig. 26. As expected, increasing the number of segments $N_{\text {seg }}$ reduces the oscillations on the spectra and improves the convergence 
of the results at low frequencies. Directivities at $r=1.5 \ell$ obtained with the RPM-FEM approach for $N_{\text {seg }}=19,39,79$ and 319 are compared with the analytical solution in Fig. 27, for frequencies $f=2.5 c_{0} / \ell, f=5 c_{0} / \ell, f=7.5 c_{0} / \ell$ and $f=10 c_{0} / \ell$. Increasing the number of time segments from 19 to 319 leads to substantial damping of the wiggles to confirm the trend toward a smooth directivity pattern.

(a)

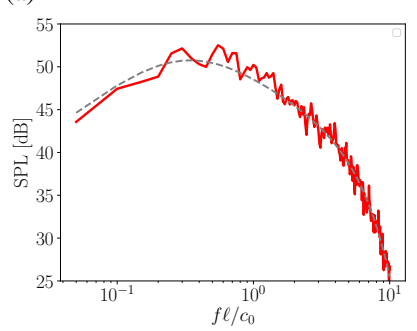

(b)

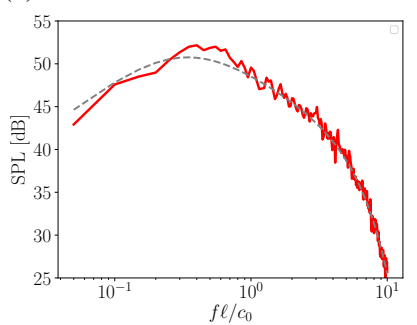

(c)

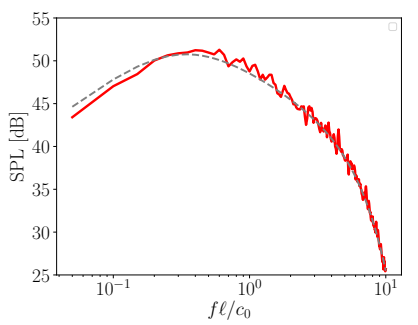

(d)

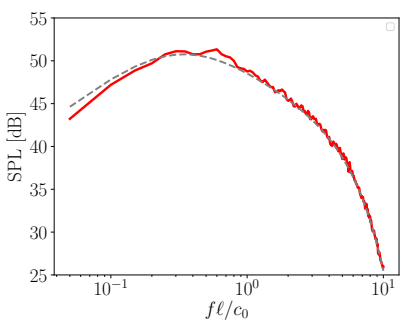

Fig. 26 SPL at $r=1.5 \ell$ and $\theta=0^{\circ}$ obtained for (a) $N_{\text {seg }}=19$, (b) $N_{\text {seg }}=39$, (c) $N_{\text {seg }}=79$ and (d) $N_{\text {seg }}=319$ : RPM-FEM and --- analytical solution.

\section{Acknowledgments}

The authors acknowledge the European Commission for its financial support through the H2020-MSCA-ITN2014 project "AEOLUS4FUTURE - Efficient harvesting of the wind energy" (Grant agreement 643167) and the UPWARDS project (Grant agreement 763990). The authors are thankful to Prof. Stéphane Moreau for providing the controlled-diffusion airfoil geometry and to Dr. Julien Christophe for providing the RANS data used for comparison.

\section{References}

[1] Roger, M., and Moreau, S., "Trailing edge noise measurements and prediction for subsonic loaded fan blades," 8 th AIAA/CEAS Aeroacoustics Conference, AIAA Paper 2002-2460, 2002. doi:https://doi.org/10.2514/6.2002-2460.

[2] Lee, S., Ayton, L., Bertagnolio, F., Moreau, S., Chong, T. P., and Joseph, P., "Turbulent boundary layer trailing-edge noise: Theory, computation, experiment, and application,” Progress in Aerospace Sciences, Vol. 126, 2021 , p. 100737. doi:https://doi.org/10.1016/j.paerosci.2021.100737.

[3] Wang, M., Moreau, S., Iaccarino, G., and Roger, M., "LES prediction of wall-pressure fluctuations and noise of a low-speed airfoil," International Journal of Aeroacoustics, Vol. 8, No. 3, 2009, pp. 177-197. doi:https://doi.org/10.1260/147547208786940017.

[4] Wolf, W. R., and Lele, S. K., "Trailing-Edge Noise Predictions Using Compressible Large-Eddy Simulation and Acoustic Analogy," AIAA Journal, Vol. 50, No. 11, 2012, pp. 2423-2434. doi:https://doi.org/10.2514/1.J051638.

[5] Wasala, S. H., Storey, R. C., Norris, S. E., and Cater, J. E., "Aeroacoustic noise prediction for wind turbines using Large Eddy Simulation,” Journal of Wind Engineering and Industrial Aerodynamics, Vol. 145, 2015, pp. 17-29. doi: https://doi.org/10.1016/j.jweia.2015.05.011. 

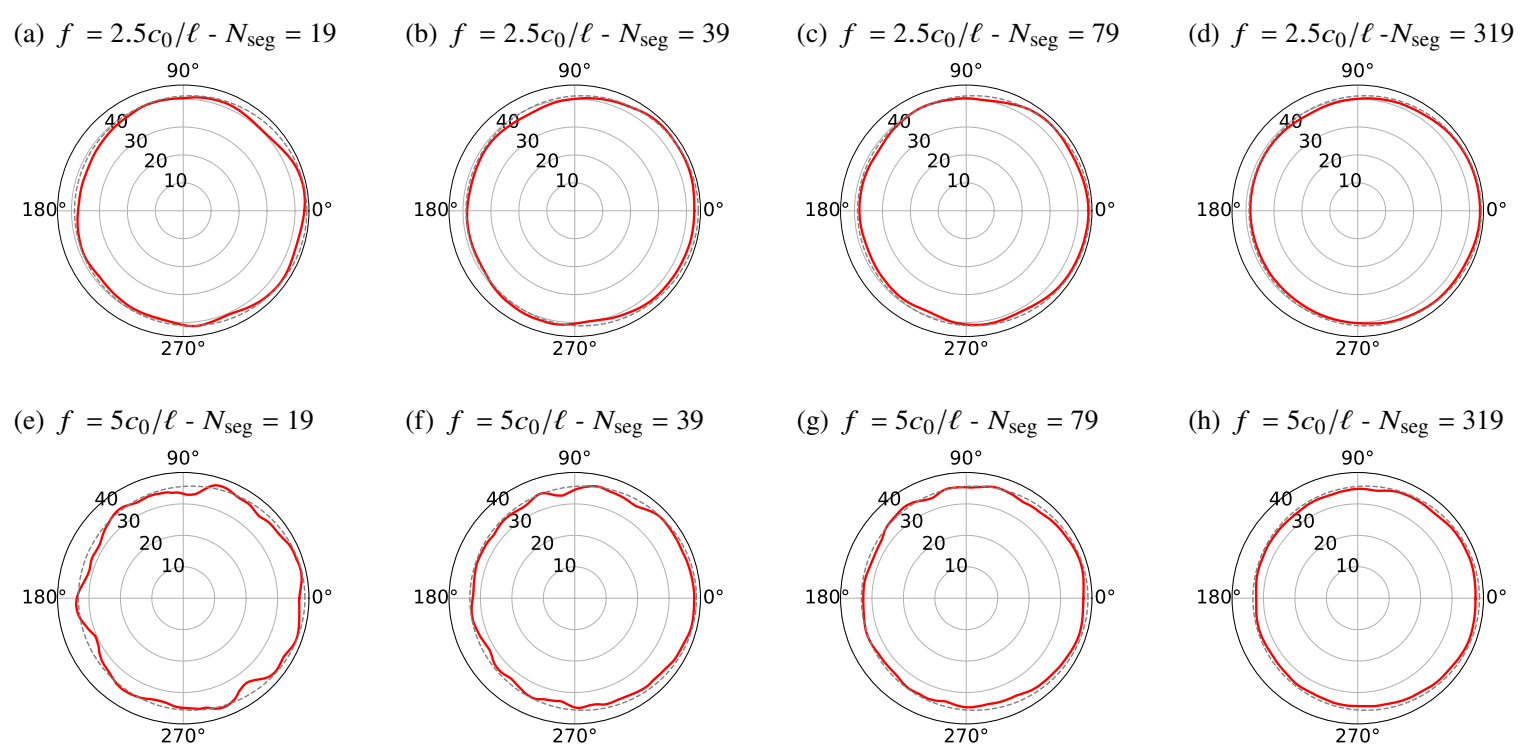

(i) $f=7.5 c_{0} / \ell-N_{\text {seg }}=19$
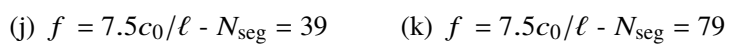

(1) $f=7.5 c_{0} / \ell-N_{\text {seg }}=319$
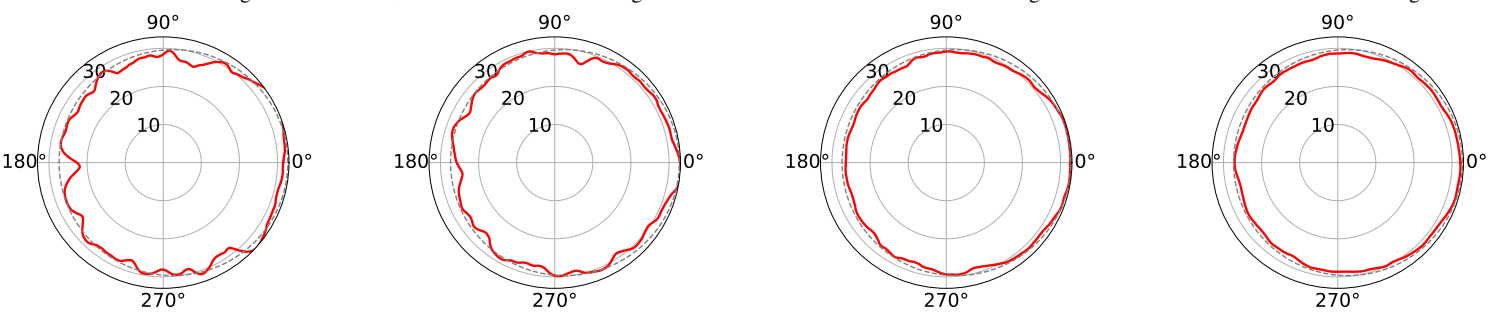

(m) $f=10 c_{0} / \ell-N_{\text {seg }}=19$

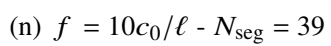

(o) $f=10 c_{0} / \ell-N_{\mathrm{seg}}=79$
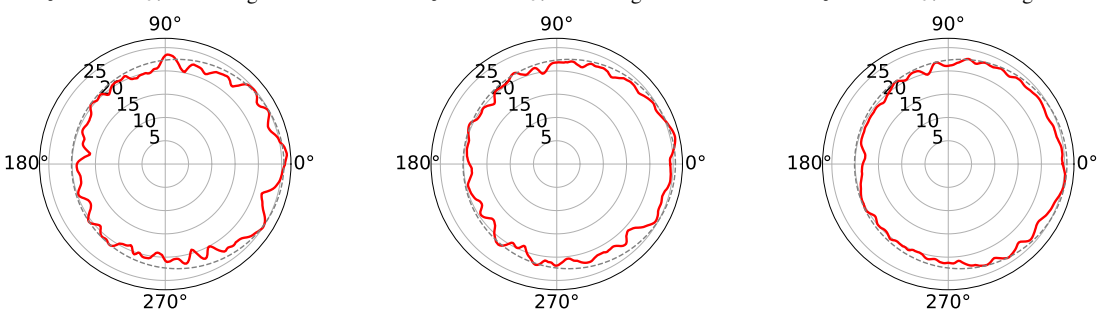

(p) $f=10 c_{0} / \ell-N_{\text {seg }}=319$

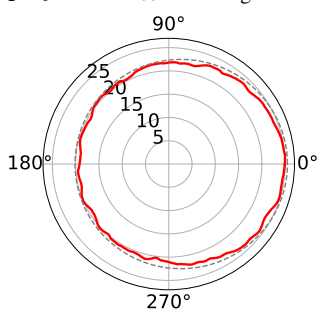

Fig. 27 Directivities of the SPL at $r=1.5 \ell$ for frequencies $2.5 c_{0} / \ell, 5 c_{0} / \ell, 7.5 c_{0} / \ell$ and $10 c_{0} / \ell$ and time segments $N_{\text {seg }}=19, N_{\text {seg }}=39, N_{\text {seg }}=79$ and $N_{\text {seg }}=319:-$ RPM-FEM and --- analytical solution.

[6] Ewert, R., and Edmunds, R., "CAA slat noise studies applying stochastic sound sources based on solenoidal digital filters," 11th AIAA/CEAS Aeroacoustics Conference, AIAA Paper 2005-2862, 2005. doi:https://doi.org/10.2514/6.2005-2862.

[7] Morris, P., and Farassat, F., "Acoustic Analogy and Alternative Theories for Jet Noise Prediction," AIAA Journal, Vol. 40, 2002, pp. 671-680. doi:https://doi.org/10.2514/2.1699.

[8] Lighthill, M. J., "On sound generated aerodynamically I. General theory," Proceedings of the Royal Society A: Mathematical, Physical and Engineering Sciences, Vol. 211, No. 1107, 1952, pp. 564-587. doi:https://doi.org/10.1098/rspa.1952.0060.

[9] Khavaran, A., and Bridges, J., "Modelling of fine-scale turbulence mixing noise," Journal of Sound and Vibration, Vol. 279, No. 3-5, 2005, pp. 1131-1154. doi:https://doi.org/10.1016/j.jsv.2003.11.054. 
[10] Kraichnan, R. H., “Diffusion by a Random Velocity Field,” Physics of Fluids, Vol. 13, No. 1, 1970, p. 22. doi:https: //doi.org/10.1063/1.1692799.

[11] Béchara, W., Bailly, C., Lafon, P., and Candel, S. M., "Stochastic Approach to Noise Modeling for Free Turbulent Flows," AIAA Journal, Vol. 32, No. 3, 1994, pp. 455-463. doi:https://doi.org/10.2514/3.12008.

[12] Bailly, C., and Juvé, D., “A Stochastic Approach To Compute Subsonic Noise Using Linearized Euler's Equations,” 5th AIAA/CEAS Aeroacoustics Conference, AIAA Paper 1999-1872, 1999. doi:https://doi.org/10.2514/6.1999-1872.

[13] Ewert, R., "Broadband slat noise prediction based on CAA and stochastic sound sources from a fast random particle-mesh (RPM) method," Computers and Fluids, Vol. 37, No. 4, 2008, pp. 369-387. doi:https://doi.org/10.1016/j.compfluid.2007.02.003.

[14] Casalino, D., and Barbarino, M., "Stochastic Method for Airfoil Self-Noise Computation in Frequency Domain,” AIAA Journal, Vol. 49, No. 11, 2011, pp. 2453-2469. doi:https://doi.org/10.2514/1.J050773.

[15] Lafitte, A., Garrec, T. L., Bailly, C., and Laurendeau, E., "Turbulence generation from a sweeping-based stochastic model," AIAA Journal, Vol. 52, No. 2, 2014, pp. 281-292. doi:https://doi.org/10.2514/1.J052368.

[16] Careta, A., Sagués, F., and Sancho, J. M., "Stochastic Generation of Homogeneous Isotropic Turbulence with Well-defined Spectra," Physical Review E, Vol. 48, No. 3, 1993, pp. 2279-2287. doi:10.1103/physreve.48.2279.

[17] Klein, M., Sadiki, A., and Janicka, J., “A digital filter based generation of inflow data for spatially developing direct numerical or large eddy simulations," Journal of Computational Physics, Vol. 186, No. 2, 2003, pp. 652-665. doi: https://doi.org/10.1016/S0021-9991(03)00090-1.

[18] Ewert, R., "RPM - the fast Random Particle-Mesh method to realize unsteady turbulent sound sources and velocity fields for CAA applications," 13th AIAA/CEAS Aeroacoustics Conference, AIAA Paper 2007-3506, 2007. doi:https://doi.org/10.2514/6.20073506.

[19] Ewert, R., Appel, C., Dierke, J., and Herr, M., "RANS/CAA Based Prediction of NACA 0012 Broadband Trailing Edge Noise and Experimental Validation,” 15th AIAA/CEAS Aeroacoustics Conference, AIAA Paper 2009-3269, 2009. doi: https://doi.org/10.2514/6.2009-3269.

[20] Ewert, R., Dierke, J., Siebert, J., Neifeld, A., Appel, C., Siefert, M., and Kornow, O., "CAA broadband noise prediction for aeroacoustic design," Journal of Sound and Vibration, Vol. 330, No. 17, 2011, pp. 4139-4160. doi:https://doi.org/10.1016/j.jsv. 2011.04.014.

[21] Cozza, I. F., Iob, A., and Arina, R., "Broadband trailing-edge noise prediction with a stochastic source model," Computers and Fluids, Vol. 57, 2012, pp. 98-109. doi:https://doi.org/10.1016/j.compfluid.2011.12.011.

[22] Proskurov, S., Darbyshire, O., and Karabasov, S., "Aerofoil broadband and tonal noise modelling using stochastic sound sources and incorporated large scale fluctuations," Journal of Sound and Vibration, Vol. 411, 2017, pp. 60-74. doi: https://doi.org/10.1016/j.jsv.2017.08.036. 
[23] Siefert, M., and Ewert, R., "Sweeping sound generation in jets realized with a random particle-mesh method," 15th AIAA/CEAS Aeroacoustics Conference (30th AIAA Aeroacoustics Conference), AIAA Paper 2009-3369, 2009. doi:https: //doi.org/10.2514/6.2009-3369.

[24] Wohlbrandt, A., Kissner, C., and Guérin, S., "Impact of cyclostationarity on fan broadband noise prediction," Journal of Sound and Vibration, Vol. 420, 2018, pp. 142-164. doi:https://doi.org/10.1016/j.jsv.2018.01.039.

[25] Allan, M. R., and Darbyshire, O. R., "Comparison of LES and Stochastic Source Generation Methods for Aero- and Hydro-Acoustic Design Guidance,” 20th AIAA/CEAS Aeroacoustics Conference, AIAA Paper 2014-2319, 2014. doi: https://doi.org/10.2514/6.2014-2319.

[26] Rautmann, C., Dierke, J., Ewert, R., Hu, N., and Delfs, J., "Generic Airfoil Trailing-Edge Noise Prediction using Stochastic Sound Sources from Synthetic Turbulence," 20th AIAA/CEAS Aeroacoustics Conference, AIAA Paper 2014-3298, 2014. doi:https://doi.org/10.2514/6.2014-3298.

[27] Ewert, R., and Schröder, W., "Acoustic perturbation equations based on flow decomposition via source filtering," Journal of Computational Physics, Vol. 188, No. 2, 2003, pp. 365-398. doi:https://doi.org/10.1016/S0021-9991(03)00168-2.

[28] Colonius, T., and Lele, S., "Computational aeroacoustics: progress on nonlinear problems of sound generation," Progress in Aerospace Sciences, Vol. 40, No. 6, 2004, pp. 345-416. doi:https://doi.org/10.1016/j.paerosci.2004.09.001.

[29] Amestoy, P. R., Duff, I. S., Koster, J., and L’Excellent, J.-Y., “A Fully Asynchronous Multifrontal Solver Using Distributed Dynamic Scheduling," SIAM Journal on Matrix Analysis and Applications, Vol. 23, No. 1, 2001, pp. 15-41. doi:https: //doi.org/10.1137/S0895479899358194.

[30] Bériot, H., Prinn, A., and Gabard, G., "Efficient implementation of high-order finite elements for Helmholtz problems," International Journal for Numerical Methods in Engineering, Vol. 106, No. 3, 2016, pp. 213-240. doi:https://doi.org/10.1002/ nme.5172.

[31] Ewert, R., Neifeld, A., and Fritzsch, A., "A 3-D modal stochastic jet noise source model," 17th AIAA/CEAS Aeroacoustics Conference, AIAA Paper 2011-2887, 2011. doi:https://doi.org/10.2514/6.2011-2887.

[32] Moreau, S., and Roger, M., "Effect of Airfoil Aerodynamic Loading on Trailing Edge Noise Sources," AIAA Journal, Vol. 43, No. 1, 2005, pp. 41-52. doi:https://doi.org/10.2514/1.5578.

[33] Moreau, S., Neal, D., and Foss, J., "Hot-Wire Measurements Around a Controlled Diffusion Airfoil in an Open-Jet Anechoic Wind Tunnel," Journal of Fluids Engineering, Vol. 128, No. 4, 2006, pp. 699-706. doi:https://doi.org/10.1115/1.2201644.

[34] Christophe, J., “Application of Hybrid Methods to High Frequency Aeroacoustics,” Ph.D. thesis, Université libre de Bruxelles, 2011.

[35] Powell, A., "Theory of vortex sound," The Journal of the Acoustical Society of America, Vol. 36, No. 1, 1964, pp. 177-195. doi:https://doi.org/10.1121/1.1918931. 
[36] Howe, M., "Contributions to the theory of aerodynamic sound, with application to excess jet noise and the theory of the flute," Journal of Fluid Mechanics, Vol. 71, No. 4, 1975, pp. 625-673. doi:https://doi.org/10.1017/S0022112075002777.

[37] Proskurov, S., Darbyshire, O., and Karabasov, S., "Aerofoil broadband and tonal noise modelling using stochastic sound sources and incorporated large scale fluctuations," Journal of Sound and Vibration, Vol. 411, 2017, pp. 60-74. doi: https://doi.org/10.1016/j.jsv.2017.08.036.

[38] Wang, M., and Moin, P., “Computation of trailing-edge flow and noise using large-eddy simulation,” AIAA Journal, Vol. 38, No. 12, 2000, pp. 2201-2209. doi:https://doi.org/10.2514/2.895.

[39] Beriot, H., and Modave, A., "An automatic perfectly matched layer for acoustic finite element simulations in convex domains of general shape," International Journal for Numerical Methods in Engineering, Vol. 122, No. 5, 2021, pp. 1239-1261. doi:https://doi.org/10.1002/nme.6560.

[40] Bermúdez, A., Hervella-Nieto, L., Prieto, A., Rodrı, R., et al., “An optimal perfectly matched layer with unbounded absorbing function for time-harmonic acoustic scattering problems," Journal of Computational Physics, Vol. 223, No. 2, 2007, pp. 469-488. doi:https://doi.org/10.1016/j.jcp.2006.09.018.

[41] Solin, P., Segeth, K., and Dolezel, I., Higher-Order Finite Element Methods, Chapman \& Hall/CRC, 2004.

[42] Lieu, A., Gabard, G., and Bériot, H., "A comparison of high-order polynomial and wave-based methods for Helmholtz problems," Journal of Computational Physics, Vol. 321, 2016, pp. 105-125. doi:https://doi.org/10.1016/j.jcp.2016.05.045.

[43] Bériot, H., and Gabard, G., "Anisotropic adaptivity of the p-FEM for time-harmonic acoustic wave propagation,” Journal of Computational Physics, Vol. 378, 2019, pp. 234-256. doi:https://doi.org/10.1016/j.jcp.2018.11.013.

[44] Caro, S., Detandt, Y., Manera, J., Mendonça, F., and Toppinga, R., "Validation of a New Hybrid CAA strategy and Application to the Noise Generated by a Flap in a Simplified HVAC Duct," 15th AIAA/CEAS Aeroacoustics Conference, AIAA Paper 2009-3352, 2009. doi:https://doi.org/10.2514/6.2009-3352.

[45] Hesthaven, J. S., and Warburton, T., Nodal Discontinuous Galerkin Methods, Texts in Applied Mathematics, Vol. 54, Springer New York, New York, NY, 2008.

[46] Dieste, M., and Gabard, G., "Random Particle Methods Applied to Broadband Fan Interaction Noise," Journal of Computational Physics, Vol. 231, No. 24, 2012, pp. 8133-8151. doi:https://doi.org/10.1016/j.jcp.2012.07.044.

[47] Menter, F. R., "Two-equation eddy-viscosity turbulence models for engineering applications," AIAA Journal, Vol. 32, No. 8, 1994, pp. 1598-1605. doi:https://doi.org/10.2514/3.12149.

[48] Ewert, R., "Slat noise trend predictions using CAA with stochastic sound sources from a random particle mesh method (RPM)," 12th AIAA/CEAS Aeroacoustics Conference, AIAA paper 2006-2667, 2006. doi:https://doi.org/10.2514/6.2006-2667.

[49] Pope, S. B., Turbulent flows, Cambridge University Press, 2000. 
[50] Ewert, R., and Dierke, J., "Stochastic Approaches for Airframe Noise Prediction,” Lecture Series 2013-03: Accurate and Efficient Aeroacoustic prediction Approaches for Airframe Noise, edited by E. L. C. Schram, R. Dénos, von Karman Institute for Fluid Dynamics, 2013, pp. 1-69.

[51] Bogey, C., and Bailly, C., "A family of low dispersive and low dissipative explicit schemes for flow and noise computations," Journal of Computational Physics, Vol. 194, No. 1, 2004, pp. 194-214. doi:https://doi.org/10.1016/j.jcp.2003.09.003.

[52] Dieste, M., "Random-vortex-particle methods applied to broadband fan interaction noise," Ph.D. thesis, University of Southampton, 2011.

[53] Geuzaine, C., and Remacle, J.-F., "Gmsh: a three-dimensional finite element mesh generator with built-in pre- and postprocessing facilities," International Journal for Numerical Methods in Engineering, Vol. 79, No. 11, 2009 , pp. 1309-1331. doi:https://doi.org/10.1002/nme.2579.

[54] Herr, M., and Kamruzzaman, M., "Benchmarking of Trailing-Edge Noise Computations—Outcome of the BANC-II Workshop," 19th AIAA/CEAS Aeroacoustics Conference, AIAA Paper 2013-2123, 2013. doi:https://doi.org/10.2514/6.2013-2123.

[55] Rautmann, C., "Numerical Simulation Concept for Low-Noise Wind Turbine Rotors," Ph.D. thesis, Technical University of Braunschweig, 2017.

[56] Gea-Aguilera, F., Gill, J., and Zhang, X., "Synthetic turbulence methods for computational aeroacoustic simulations of leading edge noise," Computers and Fluids, Vol. 157, 2017, pp. 240-252. doi:https://doi.org/10.1016/j.compfluid.2017.08.039.

[57] Amidror, I., "Scattered data interpolation methods for electronic imaging systems: a survey," Journal of Electronic Imaging, Vol. 11, No. 2, 2002, pp. 157-176. doi:https://doi.org/10.1117/1.1455013.

[58] Scott, J., Pilon, A., Lyrintzis, A., and Rozmajzl, T., "A numerical investigation of noise from a rectangular jet," 35th Aerospace Sciences Meeting and Exhibit, AIAA Paper 1997-0285, 1997. doi:https://doi.org/10.2514/6.1997-285.

[59] Gloerfelt, X., Bailly, C., and Juvé, D., "Direct computation of the noise radiated by a subsonic cavity flow and application of integral methods," Journal of Sound and Vibration, Vol. 266, No. 1, 2003, pp. 119-146. doi:https://doi.org/10.1016/S0022460X(02)01531-6.

[60] Moreau, S., Henner, M., Iaccarino, G., Wang, M., and Roger, M., “Analysis of Flow Conditions in Freejet Experiments for Studying Airfoil Self-Noise,” AIAA Journal, Vol. 41, No. 10, 2003, pp. 1895-1905. doi:https://doi.org/10.2514/2.1905.

[61] Douglas, N., "The effects of rotation on the flow field over a Controlled-Diffusion airfoil," Ph.D. thesis, Michigan State University, 2010. 